\title{
ON THE QUANTIZATION OF POLYGON SPACES*
}

\author{
L. CHARLES ${ }^{\dagger}$
}

\begin{abstract}
Moduli spaces of polygons have been studied since the nineties for their topological and symplectic properties. Under generic assumptions, these are symplectic manifolds with natural global action-angle coordinates. This paper is concerned with the quantization of these manifolds and of their action coordinates. Applying the geometric quantization procedure, one is lead to consider invariant subspaces of a tensor product of irreducible representations of $S U(2)$. These quantum spaces admit natural sets of commuting observables. We prove that these operators form a semiclassical integrable system, in the sense that they are Toeplitz operators with principal symbol the square of the action coordinates. As a consequence, the quantum spaces admit bases whose vectors concentrate on the Lagrangian submanifolds of constant action. The coefficients of the change of basis matrices can be estimated in terms of geometric quantities. We recover this way the already known asymptotics of the classical $6 j$-symbols.
\end{abstract}

Key words. Polygon space, Geometric quantization, Toeplitz operators, Lagrangian section, Symplectic reduction, $6 j$-symbol, Canonical base.

AMS subject classifications. 47L80, 53D30, 53D12, 53D50, 53D20, 81S10, 81S30, 81R12, $81 \mathrm{Q} 20$

1. Introduction. Given an $n$-tuple $\ell=\left(\ell_{1}, \ldots, \ell_{n}\right)$ of positive numbers, the polygon space $\mathcal{M}_{\ell}$ consists of the spatial $n$-sided polygons with side lengths equal to the $\ell_{i}$, up to isometries. When $\ell$ satisfies a generic assumption, $\mathcal{M}_{\ell}$ is a compact Kähler manifold. These moduli spaces have been studied in several papers since the nineties for their topology and symplectic properties [14], [11], [15] and [8]. Among other things, Kapovich and Millson discovered in [14] a remarkable action-angle coordinate system. Considering the triangulation in figure 1 , the actions are defined as the lengths of the internal edges and the angles as the dihedral angles between the faces adjacent to the internal edges. More generally, given any decomposition of a $n$-sided polygon into triangles obtained by connecting the vertices, one defines a canonical action-angle coordinate system.

The main subject of this paper is the quantum counterpart of these coordinate systems. Assuming that the lengths $\ell_{i}$ are integral, one may apply the geometric quantization procedure to the polygon space $\mathcal{M}_{\ell}$. The quantum space is defined as the space of holomorphic sections of a prequantization line bundle with base $\mathcal{M}_{\ell}$. Let $V_{m}$ be the $(m+1)$-dimensional irreducible representation of $S U(2)$ and consider the invariant subspace $\mathcal{H}_{\ell}$ of the tensor product of the $V_{\ell_{i}}$ :

$$
\mathcal{H}_{\ell}:=\left(V_{\ell_{1}} \otimes V_{\ell_{2}} \otimes \ldots \otimes V_{\ell_{n}}\right)^{S U(2)} .
$$

Then the quantum space associated to $\mathcal{M}_{\ell}$ is isomorphic to $\mathcal{H}_{\ell}$. If we replace the prequantum bundle by its $k$-th power and twist it by a half-form bundle, we obtain a quantum space isomorphic to $\mathcal{H}_{k \ell-1}$. The semi-classical limit is defined as the limit $k \rightarrow \infty$, the parameter $k$ corresponding to the inverse of the Planck constant.

The usual tools of microlocal analysis have been introduced in the context of compact Kähler manifolds and may be applied to the quantization of the polygon spaces. In particular there exists a class of operators, called Toeplitz operators, which

\footnotetext{
* Received July 10, 2009; accepted for publication January 22, 2010.

${ }^{\dagger}$ Université Pierre et Marie Curie, UMR 7586 Institut de Mathématiques de Jussieu, Paris, F75005 France (charles@math.jussieu.fr).
} 


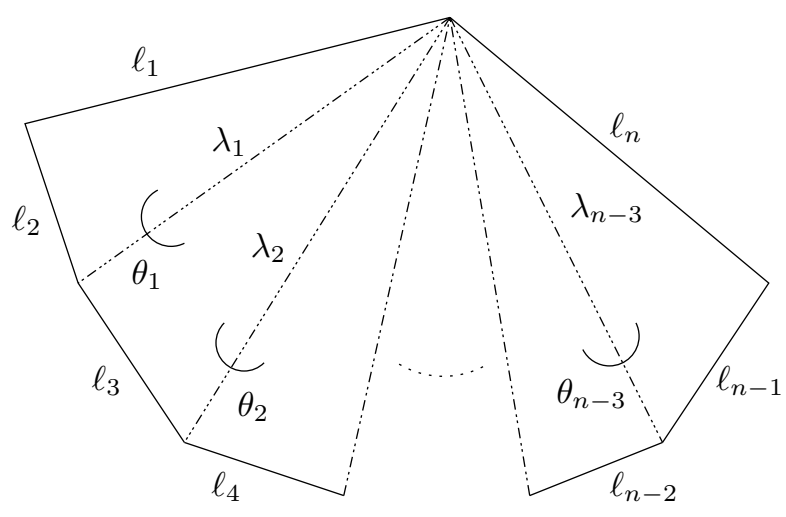

FIG. 1. Action-angle coordinates $\left(\lambda_{i}, \theta_{i}\right)_{i=1, \ldots, n-3}$.

plays a role similar to the class of pseudo-differential operators with small parameter ([2], [3]). Also relevant to this paper are the Lagrangian sections ([1], [4]). These are families of sections which in the semiclassical limit concentrate on a Lagrangian submanifold in a precise way. They are similar to the usual Lagrangian distributions of microlocal analysis.

Let us return to the vector space $\mathcal{H}_{\ell}$. Since the Littlewood-Richardson coefficients of $S U(2)$ are 0 or 1 , to each bracketing of the product $V_{\ell_{1}} \otimes \ldots \otimes V_{\ell_{n}}$ corresponds a decomposition of $\mathcal{H}_{\ell}$ into a direct sum of lines. By using concrete irreducible representations, we can even introduce well-defined bases of $\mathcal{H}_{\ell}$. In particular, the classical $6 j$-symbols are defined as the coefficients of the change of basis matrix between basis coming from $\left(\left(V_{\ell_{1}} \otimes V_{\ell_{2}}\right) \otimes V_{\ell_{3}}\right) \otimes V_{\ell_{4}}$ and $\left(V_{\ell_{1}} \otimes\left(V_{\ell_{2}} \otimes V_{\ell_{3}}\right)\right) \otimes V_{\ell_{4}}$.

For each way of placing bracket, we will define a family $\left(H_{i, \ell}\right)_{i=1, \ldots, n-3}$ of mutually commuting operators of $\mathcal{H}_{\ell}$ whose joint eigenspaces are the summand of the associated decomposition. Our main result says that the sequences $\left(H_{i, k \ell-1}\right)_{k}$ are Toeplitz operators of $\mathcal{M}_{\ell}$, cf. theorem 3.2. Further, their principal symbols are the squares of the actions of a canonical coordinate system defined as above. For instance, the parenthesising

$$
\left(\left(\ldots\left(\left(V_{\ell_{1}} \otimes V_{\ell_{2}}\right) \otimes V_{\ell_{3}}\right) \ldots\right) \otimes V_{\ell_{n-1}}\right) \otimes V_{\ell_{n}}
$$

corresponds to the angle coordinates defined in figure 1.

Families of pseudodifferential operators whose principal symbols form an integrable system have been the subject of many works, cf. [21] and references therein. Their joint spectrum can be computed in terms of the geometry of the integrable system by the Bohr-Sommerfeld conditions and their joint eigenstates are Lagrangian functions. These results have been extended to semi-classical integrable systems of Toeplitz operators in [4] and [5] and can therefore be applied to the operators $\left(H_{i, k \ell-1}\right)_{k}$. In this case, the Bohr-Sommerfeld conditions are not so interesting because their joint spectrum can easily be computed explicitely. On the other hand, it is a non-trivial result that the joint eigenspaces are generated by Lagrangian sections associated to the Lagrangian submanifolds of constant action. We prove this and also compute the symbol of these Lagrangian sections, cf. theorem 5.2.

As a consequence, we deduce the surprising formula of Roberts [17] which relates the asymptotics of $6 j$-symbols to the geometry of the tetrahedron, cf. theorem 7.1. As a matter of fact, the scalar product of two Lagrangian sections can be esti- 
mated in terms of their symbols when the associated Lagrangian manifolds intersect transversally.

Our main motivation to study the quantization of polygon spaces, besides being a natural application of our previous works, is the similarity with topological quantum field theory (TQFT). In this case we consider instead of $\mathcal{M}_{\ell}$ the moduli space of flat $S U(2)$-principal bundles on a surface $\Sigma$ with prescribed holonomy on the boundary. This moduli space admits natural Lagrangian fibrations associated to each decomposition of $\Sigma$ into pairs of pants [13]. The quantum space is the space of conformal blocks of $\Sigma$ and its dimension is given by the famous Verlinde formula. The manifestation of the Lagrangian fibration at the quantum level has been considered in several papers. Jeffrey and Weitsmann showed in [13] that the number of fibres satisfying a Bohr-Sommerfeld condition is given by the Verlinde formula. Taylor and Woodward conjectured in [19] that some bases of the quantum space of the four-holed two-sphere consist of Lagrangian sections. They deduced the asymptotics of the quantum $6 j$-symbols. Their heuristic argument was an inspiration for our estimation of the classical $6 j$-symbol.

Also, in the TQFT context, some operators called multicurve operators, play an important role. In particular, the operators associated to the curves of a decomposition into pair of pants pairwise commute and have one-dimensional joint eigenspaces. We conjecture that the multicurve operators are Toeplitz operators with principal symbol a holonomy function. Several pieces of evidence support this: the symbols of the product and of the commutators are given at first order by the usual product and the Poisson bracket [20]. Furthermore the trace of the operator is equivalent in the semiclassical limit to the average of its symbol [16]. We hope that our method could also apply to this context or at least that our results could clarify what we can expect.

The article is organized as follows. Section 2 is devoted to algebraic preliminaries. We introduce the decompositions of $\mathcal{H}_{\ell}$ as a direct sum of lines and the associated famillies of mutually commuting operators. Next section concerns the geometric quantization of the polygon spaces. Applying the "quantization commutes with reduction" theorem of Guillemin and Sternberg [9], we prove that it is isomorphic to $\mathcal{H}_{\ell}$. We also state the central result that the sequences $\left(H_{i, k \ell-1}\right)$ are Toeplitz operators. The main part of the proof is postponed to the last section of the paper. Section 4 is devoted to the symplectic geometry of the polygon spaces. Generalizing the result of [14], we associate to any triangle decomposition an action-angle coordinate system. We also describe carefully the image of the action coordinates and the associated torus actions. In section 5 , we deduce that the joint eigenstates of the $\left(H_{i, k \ell-1}\right)$ are Lagrangian sections. We also state the Bohr-Sommerfeld conditions. Section 6 is concerned with the asymptotics of the scalar product of two Lagrangian sections. At first order, it is given by a geometric pairing between the symbols of the Lagrangian sections. This is then applied in section 7 to estimate the classical $6 j$-symbols. The main part of the proof was already understood by Woodward and Taylor [19], except for the delicate phase determination. In the last section we consider the symplectic reduction of Toeplitz operators. We compute the principal and subprincipal symbols of a reduced Toeplitz operator. These subprincipal estimates are the most difficult results of the paper.

Acknowledgment. I would like to thank Julien Marché for many helpful discussions and his interest in this work. 
2. On the space $\mathcal{H}_{\ell}$.

2.1. Outline of the following sections. In section 2.2 , we introduce some notations and a class of graphs called admissible graphs. To each admissible graph will be associated a set of action coordinates of the polygon space $\mathcal{M}_{\ell}$ and also a set of mutually commuting operators of the Hilbert space $\mathcal{H}_{\ell}$. So these graphs will be used to relate the choice of a parenthesising of the product $V_{\ell_{1}} \otimes \ldots \otimes V_{\ell_{n}}$ with the choice of a decomposition of a polygon into triangles. In the analogy with the moduli space of connections and the topological quantum field theory, the admissible graphs correspond to the graphs associated to the decomposition into pairs of pants of a surface.

In section 2.3 we explain how each parenthesising of $V_{\ell_{1}} \otimes \ldots \otimes V_{\ell_{n}}$ leads to a decomposition of $\mathcal{H}_{\ell}$ as a direct sum of lines. In the next section we define for each admissible graph a set of mutually commuting operators of $\mathcal{H}_{\ell}$. Their joint spectrum is explicitly computed and their joint eigenspaces are the lines of the decomposition associated to a particular parenthesising. In the last section we show a first relationship between the spaces $\mathcal{H}_{\ell}$ and the polygons, namely that the existence of a non-vanishing vector in $\mathcal{H}_{\ell}$ is equivalent to a parity condition and the existence of a $n$-sided polygon with side lengths $\ell_{1}, \ldots, \ell_{n}$.

2.2. Notations. Our normalisation for the invariant scalar product of $\mathfrak{s u}(2)$ is

$$
\langle\xi, \eta\rangle=-\frac{1}{2} \operatorname{tr}(\xi \eta)
$$

where we identify $\mathfrak{s u}(2)$ with the space of skew-Hermitian endomorphisms of $\mathbb{C}^{2}$. As in the introduction, for any non-negative integer $m$, we denote by $V_{m}$ the irreducible representation of $S U(2)$ with spin $m / 2$, i.e. the dimension of $V_{m}$ is $m+1$. For any $n$-tuple $\ell=\left(\ell_{i}\right)_{i=1, \ldots, n}$ of non-negative integers, $\mathcal{H}_{\ell}$ is the vector space

$$
\mathcal{H}_{\ell}:=\operatorname{Inv}\left(V_{\ell_{1}} \otimes V_{\ell_{2}} \otimes \ldots \otimes V_{\ell_{n}}\right) .
$$

Let us introduce some notations regarding graphs. We say that a graph $\Gamma$ is admissible if it is connected, acyclic and trivalent. Edges with only one endpoint are permitted and called half-edges. The other edges have two endpoints and are called internal edges. We denote by $E_{\text {int }}(\Gamma)$ the set of internal edges, by $E_{\text {half }}(\Gamma)$ the set of half-edges and by $E(\Gamma)=E_{\text {int }}(\Gamma) \cup E_{\text {half }}(\Gamma)$ the set of edges. We always assume that the half-edges are numbered. Abusing notations, we often identify $E_{\text {half }}(\Gamma)$ with $\{1, \ldots, n\}$, where $n$ is the number of half-edges.

A coloring of $\Gamma$ is an assignment of a non-negative integer to each edge of $\Gamma$. We say that three non-negative integers $m, \ell$ and $p$ satisfy the Clebsch-Gordan condition $\mathrm{CG}(p, \ell, m)$ if the following holds

$$
p+\ell+m \in 2 \mathbb{Z}, \quad p \leqslant \ell+m, \quad \ell \leqslant m+p, \quad m \leqslant \ell+p .
$$

A coloring of $\Gamma$ is called admissible if for any vertex $t$, the colors of the edges incident to $t$ satisfy the Clebsch-Gordan condition.

2.3. Natural decompositions of $\mathcal{H}_{\ell}$. Let us recall that the multiplicity spaces of $S U(2)$ are one or zero-dimensional according to the Clebsch-Gordan condition (3),

$$
\operatorname{dim} \operatorname{Hom}_{S U(2)}\left(V_{k}, V_{\ell} \otimes V_{m}\right)=\left\{\begin{array}{l}
1 \text { if } \mathrm{CG}(k, \ell, m) \text { holds } \\
0 \text { otherwise }
\end{array} .\right.
$$



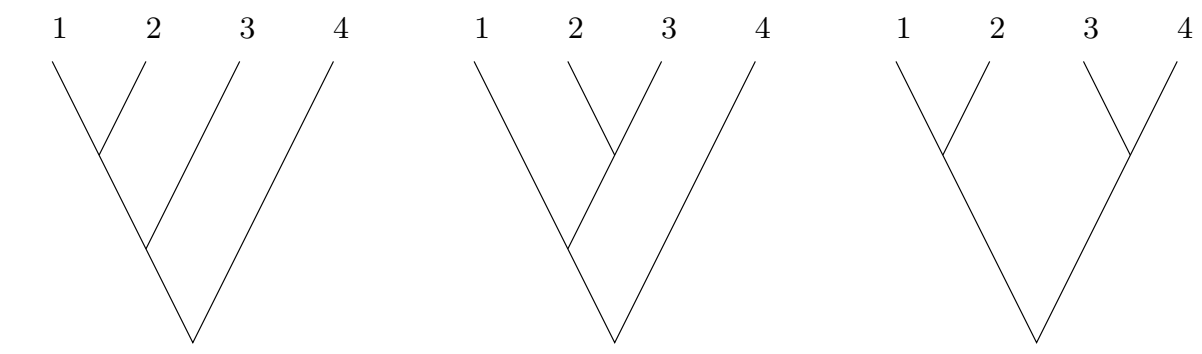

$\left(\left(V_{\ell_{1}} \otimes V_{\ell_{2}}\right) \otimes V_{\ell_{3}}\right) \otimes V_{\ell_{4}}$

$\left(V_{\ell_{1}} \otimes\left(V_{\ell_{2}} \otimes V_{\ell_{3}}\right)\right) \otimes V_{\ell_{4}}$

$\left(V_{\ell_{1}} \otimes V_{\ell_{2}}\right) \otimes\left(V_{\ell_{3}} \otimes V_{\ell_{4}}\right)$

FIG. 2. The graphs associated to various parenthesisings

So the decomposition into isotypical spaces of the tensor product of two irreducible representation is given by

$$
V_{\ell} \otimes V_{m}=\bigoplus_{k} \operatorname{Hom}_{S U(2)}\left(V_{k}, V_{\ell} \otimes V_{m}\right) \otimes V_{k}=\bigoplus_{k / C G(k, \ell, m)} V_{k} .
$$

Given a parenthesising of the tensor product of $n$ irreducible representations, one may decompose the full product into irreducible representations by applying (3) $(n-1)$ times in the order prescribed by the brackets. As instance

$$
\begin{aligned}
& V_{\ell_{1}} \otimes\left(V_{\ell_{2}} \otimes\left(V_{\ell_{3}} \otimes V_{\ell_{4}}\right)\right)=\bigoplus_{m_{1} / \mathrm{CG}\left(m_{1}, \ell_{3}, \ell_{4}\right)} V_{\ell_{1}} \otimes\left(V_{\ell_{2}} \otimes V_{m_{1}}\right) \\
& =\bigoplus_{\substack{m_{1}, m_{2} / \mathrm{CG}\left(m_{1}, \ell_{3}, \ell_{4}\right) \\
\& \mathrm{CG}\left(m_{2}, \ell_{2}, m_{1}\right)}} V_{\ell_{1}} \otimes V_{m_{2}}=\bigoplus_{\begin{array}{c}
m_{1}, m_{2}, m_{3} / \mathrm{CG}\left(m_{1}, \ell_{3}, \ell_{4}\right) \\
\mathrm{CG}\left(m_{2}, \ell_{2}, m_{1}\right)
\end{array} \& \mathrm{CG}\left(m_{3}, \ell_{1}, m_{2}\right)} V_{m_{3}} .
\end{aligned}
$$

Then taking the invariant part we obtain a decomposition into a direct sum of lines. In the previous example, we obtain

$$
\begin{gathered}
\left(V_{\ell_{1}} \otimes\left(V_{\ell_{2}} \otimes\left(V_{\ell_{3}} \otimes V_{\ell_{4}}\right)\right)\right)^{S U(2)}=\bigoplus_{\substack{m_{1}, m_{2} / \mathrm{CG}\left(m_{1}, \ell_{3}, \ell_{4}\right) \\
\mathrm{CG}\left(m_{2}, \ell_{2}, m_{1}\right) \& \mathrm{CG}\left(0, \ell_{1}, m_{2}\right)}} V_{0} \\
=\bigoplus_{\substack{m_{1} / \mathrm{CG}\left(m_{1}, \ell_{3}, \ell_{4}\right) \\
\mathrm{CG}\left(\ell_{1}, \ell_{2}, m_{1}\right)}} V_{0}
\end{gathered}
$$

because $\mathrm{CG}\left(0, \ell_{1}, m_{2}\right)$ is equivalent to $\ell_{1}=m_{2}$. In general let us show that the lines of the decomposition are naturally indexed by admissible colorings of a certain admissible graph. First one associates to each parenthesising of a product of $n$ factors, a tree with $n$ leaves, $n-2$ trivalent vertices and one bivalent vertex (called the root), cf. figure 2 for a few examples.

A coloring of such a tree is the assignment of an integer to each edge. It is admissible if the colors of the edges incident to the root are equal and if furthermore at each trivalent vertex, the colors of the incident edges satisfy the Clebsch-Gordan condition. Now given a parenthesising and the associated tree, it is clear that the summands of the decomposition coming from the parenthesising are indexed by the admissible colorings of the tree such that the edge incident to the $k$-th leave is colored by $\ell_{k}$. 

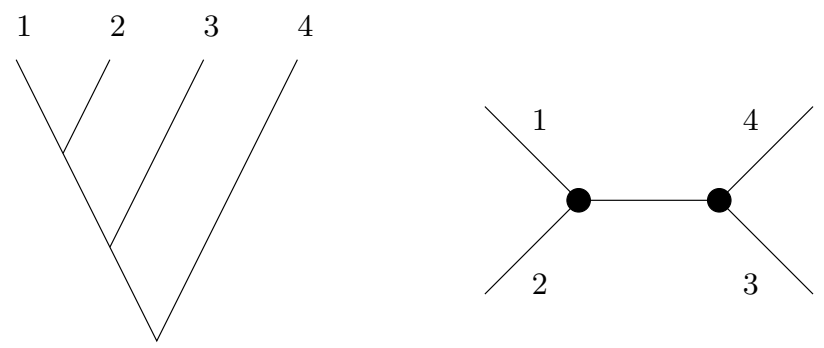

FIG. 3. A tree and its associated admissible graph

Finally given a tree as above, let us consider the admissible graph obtained by removing the leaves and the root and by merging the two edges incident to the root, cf. as instance figure 3. Observe that the admissible colorings of the graph are in one-to-one correspondence with the admissible colorings of the tree. To summarize we have proven the following

Proposition 2.1. Let $n \geqslant 3$ and $\ell$ be a n-tuple of non-negative integers. For any way of parenthesizing the product $V_{\ell_{1}} \otimes \ldots \otimes V_{\ell_{n}}$, there exists an admissible graph $\Gamma$ with $n$ half-edges and a decomposition into a sum of lines

$$
\mathcal{H}_{\ell}=\bigoplus D_{\varphi}
$$

where $\varphi$ runs over the colorings of $\Gamma$ such that $\varphi(i)=\ell_{i}$ for any half-edge $i$.

Observe that different parenthesisings may be associated to the same graph. As we will see in the next section not only the indexing set but also the summands $D_{\varphi}$ of the decomposition depend only on the graph $\Gamma$.

2.4. A complete set of observables. Recall first that the Casimir operator $Q$ of a representation $\rho$ of $S U(2)$ on a finite dimensional vector space $V$ is the self-adjoint operator $V \rightarrow V$ defined by

$$
Q=-\left(\rho\left(\xi_{1}\right)^{2}+\rho\left(\xi_{2}\right)^{2}+\rho\left(\xi_{3}\right)^{2}\right)
$$

where $\left(\xi_{1}, \xi_{2}, \xi_{3}\right)$ is an orthogonal base of $\mathfrak{s u}(2)$. Its eigenspaces are the isotypical subspaces of $V$, the eigenvalue $4 j(j+1)$ corresponding to the spin $j$ isotypical subspace.

The following lemma will be useful in the sequel. Consider a representation $\rho_{1} \times \rho_{2}$ of $S U(2) \times S U(2)$ on a finite dimensional vector space $V$. Denote by $\rho$ the $S U(2)$ representation on $V$ by the diagonal inclusion $\rho(g)=\rho_{1}(g) \times \rho_{2}(g)$.

Lemma 2.1. The Casimir operators $Q_{1}$ and $Q_{2}$ of the representations $\rho_{1}$ and $\rho_{2}$ commute with $\rho$. Furthermore the restrictions of $Q_{1}$ and $Q_{2}$ to the $\rho$-invariant part of $V$ coincide.

Proof. To check this, it suffices to decompose $V$ as the sum of isotypical subspaces for the product representation $\rho_{1} \times \rho_{2}$. Indeed $Q_{1}$ and $Q_{2}$ preserve this decomposition and act by multiplication by $a(a+2)$ and $b(b+2)$ respectively on the $V_{a} \otimes V_{b}$-isotypical subspace. Furthermore, it follows from (3) that the $\rho$-invariant part of the $V_{a} \otimes V_{b^{-}}$ isotypical subspace is trivial if $a \neq b$. 
For any subset $I$ of $\{1, \ldots, n\}$, let $\rho_{I, \ell}$ be the representation of $S U(2)$ in the tensor product $V_{\ell_{1}} \otimes \ldots \otimes V_{\ell_{n}}$ given by

$$
\rho_{I, \ell}(g)\left(v_{1} \otimes \ldots \otimes v_{n}\right)=w_{1} \otimes \ldots \otimes w_{n}, \quad \text { where } w_{i}=\left\{\begin{array}{l}
g \cdot v_{i} \text { if } i \in I \\
v_{i} \text { otherwise }
\end{array}\right.
$$

In particular $\rho_{\{1, \ldots, n\}, \ell}$ is the diagonal representation whose invariant subspace is $\mathcal{H}_{\ell}$. By the previous lemma, the Casimir operator $Q_{I, \ell}$ of $\rho_{I, \ell}$ preserves the subspace $\mathcal{H}_{\ell}$. Furthermore the restrictions of $Q_{I, \ell}$ and $Q_{I^{c}, \ell}$ to $\mathcal{H}_{\ell}$ coincide.

Consider now an admissible graph $\Gamma$ with $n$ half-edges. Let $a$ be an internal edge of $\Gamma$. Assume $a$ is directed and consider the set $I(a)$ of half-edges which are connected to the initial vertex of $a$ by a path which does not contain the terminal vertex of $a$. We define the operator

$$
H_{a, \ell}: \mathcal{H}_{\ell} \rightarrow \mathcal{H}_{\ell}
$$

as the restriction of $Q_{I(a), \ell}$ to $\mathcal{H}_{\ell}$. If we change the direction of $a$, we replace $I(a)$ by its complementary subset. So the operator $H_{a, \ell}$ is defined independently of the orientation of the edge.

TheOREM 2.1. Let $n \geqslant 4$. Let $\Gamma$ be an admissible graph with $n$ half-edges. Let $\ell$ be a $n$-tuple of non-negative integers. Then the operators $H_{a, \ell}, a \in E_{\mathrm{int}}(\Gamma)$, mutually commute. The joint eigenvalues of these operators are the $(n-3)$-tuples

$$
(\varphi(a)(\varphi(a)+2))_{a \in E_{\mathrm{int}}(\Gamma)}
$$

where $\varphi$ runs over the admissible colorings of $\Gamma$ such that $\varphi(i)=\ell_{i}$ for any half-edge $i$. Each joint eigenvalue is simple.

Furthermore if $\Gamma$ is the graph associated to a parenthesising of $V_{\ell_{1}} \otimes \ldots \otimes V_{\ell_{n}}$ as in proposition 2.1, the joint eigenspace associated to the coloring $\varphi$ is the line $D_{\varphi}$ of proposition 2.1 .

Proof. To prove that $H_{a, \ell}$ and $H_{b, \ell}$ commute for any internal edges $a$ and $b$, we use that these operators do not depend on the direction of the edges. Changing these directions if necessary, we may assume that $I(a)$ and $I(b)$ are disjoint. Then the representations $\rho_{I(a), \ell}$ and $\rho_{I(b), \ell}$ commute. So their Casimir operators also commute.

Observe that for any permutation $\sigma$ of $\{1, \ldots, n\}$, the canonical isomorphism

$$
V_{\ell_{1}} \otimes \ldots \otimes V_{\ell_{n}} \rightarrow V_{\ell_{\sigma(1)}} \otimes \ldots \otimes V_{\ell_{\sigma(n)}}
$$

intertwins $Q_{I(a), \ell}$ with $Q_{\sigma(I(a)), \sigma(\ell)}$ where $\sigma(\ell)=\left(\ell_{\sigma(1)}, \ldots, \ell_{\sigma(n)}\right)$. Hence it is sufficient to prove the first part of the theorem for one order of the half-edges. It is easily seen that the graph $\Gamma$ endowed with an appropriate order of the half-edges is induced by a parenthesising of the product $V_{\ell_{1}} \otimes \ldots \otimes V_{\ell_{n}}$. So it suffices to show that each summand $D_{\varphi}$ of the associated decomposition is the joint eigenspace of the $\left(H_{a, \ell}\right)$ with joint eigenvalue $(\varphi(a)(\varphi(a)+2))$.

To see this, one considers first the tree associated to the parenthesising and orient each edge from the leaves to the root. Then one may associate a representation $\rho_{I(a), \ell}$ to each internal edge $a$ of the tree exactly as we did for admissible graphs. Recall that to define the summands $D_{\varphi}$, we first express $V_{\ell_{1}} \otimes \ldots \otimes V_{\ell_{n}}$ as a direct sum of irreducible representations by decomposing the partial products with (3). This amounts to decompose $V_{\ell_{1}} \otimes \ldots \otimes V_{\ell_{n}}$ with respect to the isotypical subspaces of the 


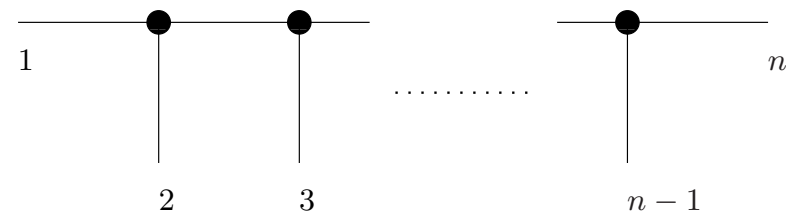

Fig. 4. The graph $\Gamma_{n}$

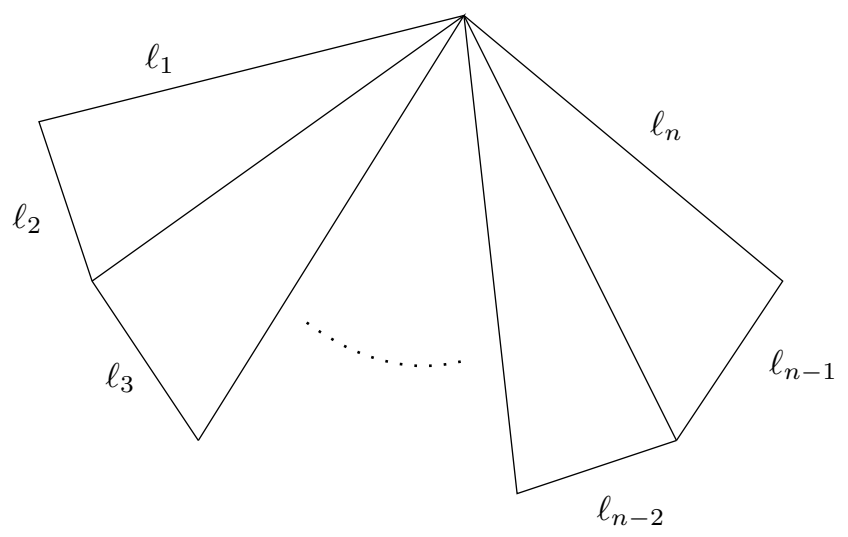

FIG. 5. The triangulation associated to $\Gamma_{n}$

$\rho_{I(a), \ell}$ and $\rho_{\{1, \ldots, n\}, \ell}$, the last one for the root. Equivalently one considers the direct sum of the joint eigenspaces of the associated Casimir operators $Q_{I(a), \ell}$ and $Q_{\{1, \ldots, n\}, \ell}$. Then we take the invariant part which is the kernel of $Q_{\{1, \ldots, n\}, \ell}$. To conclude observe that the operators $Q_{I(a), \ell}$ associated to the internal edges of the tree are the same as the ones associated to the internal edges of $\Gamma$.

2.5. On the non-triviality of $\mathcal{H}_{\ell}$. Since it is preferable to know whether $\mathcal{H}_{\ell}$ is trivial, we prove the following

Proposition 2.2. Let $n \geqslant 1$ and $\ell$ be a n-tuple of non-negative integers. Then $\mathcal{H}_{\ell}$ is not trivial if and only if the sum $\ell_{1}+\ldots+\ell_{n}$ is even and there exists a $n$-sided planar polygon with side lengths $\ell_{1}, \ldots, \ell_{n}$.

Furthermore the existence of a planar polygon with side lengths $\ell_{1}, \ldots, \ell_{n}$ is equivalent to the inequalities $\ell_{j} \leqslant \frac{1}{2}\left(\ell_{1}+\ldots+\ell_{n}\right)$ for $j=1, \ldots, n$.

Proof. If $n=1,2,3$, the result is easily proved. For $n \geqslant 3$, consider the graph $\Gamma_{n}$ in figure 4. By proposition 2.1, the non-triviality of $\mathcal{H}_{\ell}$ is equivalent to the existence of a coloring $\varphi$ of $\Gamma_{n}$ such that $\varphi(i)=\ell_{i}$ for any $i$. If such a coloring exists, by summing the parity conditions (2) at each vertex we obtain that the sum of the $\ell_{i}$ is even. Furthermore the inequalities in (2) are equivalent to the existence of a triangle with side lengths $k, \ell, m$. Given a coloring of $\Gamma$, one can patch together the triangles associated to the various vertices as in figure 5 . One obtains a $n$-sided polygon with the required side lengths.

For the converse, we may assume without restriction that the lengths $\ell_{i}$ do not vanish. Then the proof is by induction on $n$. For $n \geqslant 3$, consider a $(n+1)$-tuple $\left(\ell_{i}\right)$ satisfying the parity condition and assume that there exists a family of vectors $\left(v_{i}\right)$ with lengths $\left|v_{i}\right|=\ell_{i}$ and such that the sum $v_{1}+\ldots+v_{n+1}$ vanishes. Then it suffices 
to prove that we can choose these vectors in such a way that

$$
\ell_{n}^{\prime}=\left|v_{n}+v_{n+1}\right|
$$

is integral and $\ell_{n}^{\prime}+\ell_{n}+\ell_{n+1}$ is even. Indeed, if it is the case, one may apply the induction assumption to $\left(\ell_{1}, \ldots, \ell_{n-1}, \ell_{n}^{\prime}\right)$ and we get a admissible coloring $\varphi$ of the graph $\Gamma_{n}$ with $\varphi(i)=\ell_{i}$ for $i=1, \ldots, n-1$ and $\varphi(n)=\ell_{n}^{\prime}$. Then we extend this coloring to an admissible coloring of $\Gamma_{n+1}$ by assigning $\ell_{n}$ and $\ell_{n+1}$ to the $n$-th and $(n+1)$-th half-edges.

Let us prove the existence of the $v_{i}$ satisfying the extra condition. The minimum and maximum values of $\left|v_{n}+v_{n+1}\right|$ when $v_{n}$ and $v_{n+1}$ run over the circles of radius $\ell_{n}$ and $\ell_{n+1}$ are

$$
m=\left|\ell_{n}-\ell_{n+1}\right|, \quad M=\ell_{n}+\ell_{n+1} .
$$

Similarly if $\left|v_{i}\right|=\ell_{i}$ for $i=1, \ldots, n-1$, the minimum and maximum of $\left|v_{1}+\ldots+v_{n-1}\right|$ are

$$
m^{\prime}=\max \left(2 L-\left(\ell_{1}+\ldots+\ell_{n-1}\right), 0\right), \quad M^{\prime}=\ell_{1}+\ldots+\ell_{n-1}
$$

where $L$ is the maximum of $\ell_{1}, \ldots, \ell_{n-1}$. Observe also that $m<M$ and $m^{\prime}<M^{\prime}$ because the lengths $\ell_{i}$ are positive.

Then the existence of the family $\left(v_{i}\right)$ such that $v_{1}+\ldots+v_{n+1}=0$ is equivalent to the existence of a length $\ell_{n}^{\prime} \in[m, M] \cap\left[m^{\prime}, M^{\prime}\right]$. We have to prove that if this intersection is non-empty then it contains an integer $\ell_{n}^{\prime}$ such that $\ell_{n}^{\prime}+\ell_{n}+\ell_{n+1}$ is even. Since the endpoints $m, M, m^{\prime}$ and $M^{\prime}$ are all integral, if $[m, M] \cap\left[m^{\prime}, M^{\prime}\right]$ is not empty, it contains at least two consecutive integers or it consists of one point. In this last case, one has $M=m^{\prime}$ or $m=M^{\prime}$, hence $\ell_{n}^{\prime}=m$ or $M$ which is integral and satisfies the parity condition.

3. Semi-classical properties of $\mathcal{H}_{\ell}$. Applying the geometric quantization procedure, we give a geometric construction of the spaces $\mathcal{H}_{\ell}$. We recall the general setting in a first section and explain in the next one how the spaces $\mathcal{H}_{\ell}$ are viewed as quantization of polygon spaces. In the following sections, we define Toeplitz operators and then prove that the operators $H_{a, \ell}$ introduced previously are Toeplitz operators.

3.1. Geometric quantization. Consider a compact connected symplectic manifold $(M, \omega)$. Assume that it is endowed with:

- a Kähler structure whose fundamental form is $\omega$,

- a prequantization bundle $L \rightarrow M$, that is a holomorphic Hermitian line bundle whose Chern curvature is $\frac{1}{i} \omega$,

- a half-form bundle $\delta \rightarrow M$, that is a square root of the canonical bundle of $M$.

The quantum space associated to these data is the space of holomorphic sections of $L \otimes \delta$ that we denote by $H^{0}(M, L \otimes \delta)$. It has the scalar product

$$
\left(\Psi_{1}, \Psi_{2}\right)=\int_{M}\left(\Psi_{1}(x), \Psi_{2}(x)\right)_{L_{x} \otimes \delta_{x}} \mu_{M}(x)
$$

induced by the metric of $L \otimes \delta$ and the Liouville measure $\mu_{M}=\omega^{n} / n$ !.

Consider a Hamiltonian action of a Lie group $G$ on $M$ with momentum $\mu: M \rightarrow$ $\mathfrak{g}^{*}$. By definition, the momentum is equivariant and satisfies

$$
d \mu^{\xi}+\omega\left(\xi^{\#}, .\right)=0, \quad \forall \xi \in \mathfrak{g}
$$


where $\xi^{\#}$ is the infinitesimal vector field of $M$ associated to $\xi$. We assume that the action lifts to the prequantization bundle in such a way that the infinitesimal action on the sections of $L$ is

$$
\nabla_{\xi^{\#}}^{L}+i \mu^{\xi} \quad \forall \xi \in \mathfrak{g} .
$$

Furthermore we assume that the action on $M$ preserves the complex structure and lifts to the half-form bundle. With these assumptions, the group $G$ acts naturally on $H^{0}(M, L \otimes \delta)$, the infinitesimal action being given by the Kostant-Souriau operators

$$
i \mu^{\xi}+\nabla_{\xi^{\#}}^{L} \otimes \mathrm{id}+\mathrm{id} \otimes \mathcal{L}_{\xi^{\#}}, \quad \forall \xi \in \mathfrak{g} .
$$

Here $\mathcal{L}$ is the Lie derivative of half-forms.

All the irreducible representations of compact Lie groups may be obtained in this way. Starting from the irreducible representations of $S U(2)$, we will construct the tensor product $V_{\ell_{1}} \otimes \ldots \otimes V_{\ell_{n}}$ and its invariant subspace as quantum spaces associated to some symplectic manifolds.

3.2. Geometric realization of the space $\mathcal{H}_{\ell}$. Consider the tautological bundle $\mathcal{O}(-1)$ of the projective complex line $\mathbb{P}^{1}(\mathbb{C})$ and let $\mathcal{O}(m)=\mathcal{O}(-1)^{-m}$. These are $S U(2)$-bundles with base $\mathbb{P}^{1}(\mathbb{C})$. For any integer $m \geqslant 1$, the induced $S U(2)$-action on the holomorphic sections of

$$
\mathcal{O}(m) \otimes \mathcal{O}(-1) \rightarrow \mathbb{P}^{1}(\mathbb{C})
$$

is the irreducible representation $V_{m-1}$. Denote by $\omega_{F S}$ the Fubiny-Study form of $\mathbb{P}^{1}(\mathbb{C})$. Then $\mathcal{O}(m)$ is a prequantization bundle with curvature $\frac{1}{i} m \omega_{F S}$. Furthermore $\mathcal{O}(-1)$ is the unique half-form bundle of $\mathbb{P}^{1}(\mathbb{C})$. The $S U(2)$-action satisfies all the general properties stated below. Its momentum

$$
\mu_{m}: \mathbb{P}^{1}(\mathbb{C}) \rightarrow(\mathfrak{s u}(2))^{*}
$$

is an embedding whose image is the coadjoint orbit with symplectic volume $2 \pi m$. With our normalization of the scalar product, this coadjoint orbit is the sphere $S_{m}^{2}$ with radius $m$ centered at the origin. In the following we identify $\left(\mathbb{P}^{1}(\mathbb{C}), m \omega_{F S}\right)$ with $S_{m}^{2}$.

Next we consider the product $S_{\ell_{1}}^{2} \times \ldots \times S_{\ell_{n}}^{2}$ with the prequantization bundle and the half-form bundle:

$$
\mathcal{O}\left(\ell_{1}\right) \otimes \ldots \otimes \mathcal{O}\left(\ell_{n}\right), \quad \mathcal{O}(-1) \otimes \ldots \otimes \mathcal{O}(-1) .
$$

The associated quantum space is the tensor product $V_{\ell_{1}-1} \otimes \ldots \otimes V_{\ell_{n}-1}$. The diagonal action of $S U(2)$ is Hamiltonian with momentum

$$
\mu=\pi_{1}^{*} \mu_{\ell_{1}}+\pi_{2}^{*} \mu_{\ell_{2}}+\ldots+\pi_{n}^{*} \mu_{\ell_{n}}
$$

where $\pi_{i}$ is the projection from $S_{\ell_{1}} \times \ldots \times S_{\ell_{n}}$ onto the $i$-th factor. It satisfies all the previous assumptions.

The invariant subspace of $V_{\ell_{1}-1} \otimes \ldots \otimes V_{\ell_{n}-1}$ is the Hilbert space $\mathcal{H}_{\ell-1}$ we introduced previously. It follows from the "quantization commutes with reduction" theorem proved in [9] that $\mathcal{H}_{\ell-1}$ is the quantum space associated with the symplectic quotient $\mathcal{M}_{\ell}$ of $S_{\ell_{1}}^{2} \times \ldots \times S_{\ell_{n}}^{2}$ by $S U(2)$. More precisely we assume that

$$
\ell_{j} \leqslant \frac{1}{2}\left(\ell_{1}+\ldots+\ell_{n}\right), \quad j=1, \ldots, n,
$$


and

$$
\ell_{1} \pm \ell_{2} \pm \ldots \pm \ell_{n} \neq 0
$$

for any possible choice of signs. The first assumption is equivalent to the nonemptyness of $\mathcal{M}_{\ell}$. The second one is to ensure that the quotient does not have any singularity. Indeed, (10) holds if and only if the $S U(2)$-action on the null set $\{\mu=0\}$ factorizes through a free $S O(3)$-action. So under this assumption the quotient $\mathcal{M}_{\ell}$ is a manifold. It inherits by reduction a Kähler structure. We assume furthermore that

$$
\ell_{1}+\ldots+\ell_{n} \in 2 \mathbb{Z} \text { and } n \in 2 \mathbb{Z} .
$$

Then the diagonal $S U(2)$-action on the line bundles (8) factors through a $S O(3)$ action. The quotients of the restriction at $\{\mu=0\}$ of these bundles are genuine line bundles with base $\mathcal{M}_{\ell}$, that we denote by $L_{\ell}$ and $\delta_{\ell}$ respectively. $L_{\ell}$ has a natural structure of prequantization bundle and $\delta_{\ell}$ is a half-form bundle. The restrictions at $\{\mu=0\}$ of the invariant sections descend to the quotient $\mathcal{M}_{\ell}$. This defines a map

$$
\mathcal{H}_{\ell-1} \rightarrow H^{0}\left(\mathcal{M}_{\ell}, L_{\ell} \otimes \delta_{\ell}\right)
$$

which is a vector space isomorphism. Additional details on this construction will be recalled in section 8.1. Applying the same construction to the power of the prequantization bundle, we obtain the following theorem.

THEOREM 3.1. Let $n \geqslant 4$ and $\ell=\left(\ell_{1}, \ldots, \ell_{n}\right)$ be a family of positive integers satisfying (9), (10) and (11). Then for any positive integer $k$, we have a natural vector space isomorphism

$$
V_{k, \ell}: \mathcal{H}_{k \ell-1} \rightarrow H^{0}\left(\mathcal{M}_{\ell}, L_{\ell}^{k} \otimes \delta_{\ell}\right)
$$

Since the maps $V_{k, \ell}$ do not necessarily preserve the scalar product, we will also consider the unitary operators $V_{k, \ell}\left(V_{k, \ell}^{*} V_{k, \ell}\right)^{-1 / 2}$. The asymptotic results we prove in this paper are in the limit $k \rightarrow \infty$.

REMARK 3.1. In the case where assumption (10) does not hold, one can still identify the Hilbert space $\mathcal{H}_{k \ell-1}$ with a space of holomorphic sections on a Kähler analytic space [18]. We will not consider this case because the theory of Toeplitz operators has not been developed for singular spaces. The assumption (11) is not really necessary. Actually we could extend our results to the sequences

$$
\mathcal{H}_{k \ell-1+m}, \quad k=1,2, \ldots
$$

where $\ell$ and $m$ are any multi-indices satisfying (10). Recall that $\mathcal{H}_{\varphi}$ is a trivial vector space when $|\varphi|=\varphi(1)+\ldots+\varphi(n)$ is odd. Assume that $|\ell|$ and $n$ are even. then we may consider without restriction that $|m|$ is even too. Let $K \rightarrow \mathcal{M}_{\ell}$ be the quotient bundle of the restriction at $\{\mu=0\}$ of $\mathcal{O}\left(m_{1}\right) \otimes \ldots \otimes \mathcal{O}\left(m_{n}\right)$. We have an isomorphism

$$
\mathcal{H}_{k \ell-1+m} \simeq H^{0}\left(\mathcal{M}_{\ell}, L_{\ell}^{k} \otimes K \otimes \delta_{\ell}\right) .
$$

Then we can apply the methods used in this paper as it is explained in [5] and [7]. When $|\ell|$ is even whereas $n$ and $|m|$ are odd, we can not define globally the bundles $K$ and $\delta_{\ell}$. But their tensor product is still perfectly defined. When $|\ell|$ is odd, by doing the parameter change $k=2 k^{\prime}+1$ or $k=2 k^{\prime}$, we are reduced to the previous cases. Here to avoid the complications due to the auxiliary bundle $K$, we consider uniquely the spaces $\mathcal{H}_{k \ell-1}$ under the assumption that $|\ell|$ and $n$ are even. 
3.3. Toeplitz operators. Let $(M, \omega)$ be a compact Kähler manifold with a prequantization bundle $L$ and a half-form bundle $\delta$. For any integer $k$ and any function $f \in \mathcal{C}^{\infty}(M)$, we consider the rescaled Kostant-Souriau operator (cf. (7))

$$
\mathcal{P}_{k}(f)=f+\frac{1}{i k}\left(\nabla_{X}^{L^{k}} \otimes \mathrm{id}+\mathrm{id} \otimes \mathcal{L}_{X}^{\delta}\right): \mathcal{C}^{\infty}\left(M, L^{k} \otimes \delta\right) \rightarrow \mathcal{C}^{\infty}\left(M, L^{k} \otimes \delta\right)
$$

where $X$ is the Hamiltonian vector field of $f$. These operators do not necessarily preserve the subspace of holomorphic sections. Let us consider $H^{0}\left(M, L^{k} \otimes \delta\right)$ as a subspace of the space of $L^{2}$-sections. Assume this last space is endowed with the scalar product induced by the metrics of $L, \delta$ and the Liouville form. Introduce the orthogonal projector $\Pi_{k}$ onto $H^{0}\left(M, L^{k} \otimes \delta\right)$. Then a Toeplitz operator is defined as a family of operators

$$
\left(T_{k}:=\Pi_{k} \mathcal{P}_{k}(f(\cdot, k))+R_{k}: H^{0}\left(M, L^{k} \otimes \delta\right) \rightarrow H^{0}\left(M, L^{k} \otimes \delta\right)\right)_{k=1,2, \ldots}
$$

where $(f(\cdot, k))$ is a sequence of $\mathcal{C}^{\infty}(M)$ which admits an asymptotic expansion

$$
f(\cdot, k)=f_{0}+k^{-1} f_{1}+k^{-2} f_{2}+\ldots
$$

for the $\mathcal{C}^{\infty}$-topology with $f_{0}, f_{1} \ldots \in \mathcal{C}^{\infty}(M)$. Furthermore $\left(R_{k}\right)$ is any operator such that $\left\|R_{k}\right\|=O\left(k^{-\infty}\right)$. As a result the coefficients $f_{\ell}$ are determined by $\left(T_{k}\right)$. We call $f_{0}$ the principal symbol of $\left(T_{k}\right)$ and $f_{1}$ its subprincipal symbol.

3.4. The Casimir operators. Consider now the symplectic quotient $\mathcal{M}_{\ell}$ and its quantization as in section 3.2. For any subset $I$ of $\{1, \ldots, n\}$, we denote by $q_{I, \ell}$ the function

$$
q_{I, \ell}:=\left|\sum_{i \in I} \pi_{i}^{*} \mu_{\ell_{i}}\right|^{2} \in \mathcal{C}^{\infty}\left(S_{\ell_{1}}^{2} \times \ldots \times S_{\ell_{n}}^{2}\right) .
$$

It is invariant and descends to a function $h_{I, \ell}$ of $\mathcal{C}^{\infty}\left(\mathcal{M}_{\ell}\right)$. Recall that we defined in section 2.4 a Casimir operator $Q_{I, \ell}$ which acts on the space $\mathcal{H}_{\ell}$ and that we introduced in section 3.2 isomorphisms $V_{k}$ from $\mathcal{H}_{k \ell-1}$ to $H^{0}\left(\mathcal{M}_{\ell}, L_{\ell}^{k} \otimes \delta_{\ell}\right)$. A central result of the paper is the following theorem.

TheOREM 3.2. Let $n \geqslant 4$ and $\ell=\left(\ell_{1}, \ldots, \ell_{n}\right)$ be a family of positive integers satisfying (9), (10) and (11). For any subset I of $\{1, \ldots, n\}$, the sequence

$$
\left(\frac{1}{k^{2}} V_{k} Q_{I, k \ell-1} V_{k}^{-1}: H^{0}\left(\mathcal{M}_{\ell}, L_{\ell}^{k} \otimes \delta_{\ell}\right) \rightarrow H^{0}\left(\mathcal{M}_{\ell}, L_{\ell}^{k} \otimes \delta_{\ell}\right)\right)_{k=1,2, \ldots}
$$

is a Toeplitz operator with principal symbol $h_{I, \ell}$ and vanishing subprincipal symbol.

Let us sketch the proof. First it follows from the results of [2] that the product of two Toeplitz operators $\left(T_{k}\right)$ and $\left(S_{k}\right)$ is a Toeplitz operators. The principal and subprincipal symbols $f_{0}, f_{1}$ of $\left(T_{k} S_{k}\right)$ may be computed in terms of the symbols $g_{0}, g_{1}$ of $\left(T_{k}\right)$ and $h_{0}, h_{1}$ of $\left(S_{k}\right)$

$$
f_{0}=g_{0} h_{0}, \quad f_{1}=g_{0} h_{1}+h_{0} g_{1}+\frac{1}{2 i}\left\{f_{0}, g_{0}\right\},
$$

cf. [5] for a proof of the second formula. Second, in the case of a Hamiltonian action $G$ with momentum $\mu$ which satisfies all the assumptions of section 3.1, the infinitesimal 
action of $\xi \in \mathfrak{g}$ on $H^{0}\left(M, L^{k} \otimes \delta\right)$ is the Kostant-Souriau operator $i k \mathcal{P}\left(\mu^{\xi}\right)$. Since it preserves $H^{0}\left(M, L^{k} \otimes \delta\right)$, the sequence $\left(\mathcal{P}\left(\mu^{\xi}\right)\right)_{k}$ is a Toeplitz operator. Its principal symbol is $\mu^{\xi}$ and its subprincipal symbol vanishes.

Using these two general facts, we deduce from the expression (4) of the Casimir operator that the sequence

$$
\left(\frac{1}{k^{2}} Q_{I, k \ell-1}: V_{k \ell_{1}-1} \otimes \ldots \otimes V_{k \ell_{n}-1} \rightarrow V_{k \ell_{1}-1} \otimes \ldots \otimes V_{k \ell_{n}-1}\right)_{k=1,2, \ldots}
$$

is a Toeplitz operator of $S_{\ell_{1}}^{2} \times \ldots \times S_{\ell_{n}}^{2}$. With our normalization for the scalar product of $\mathfrak{s u}(2)$, its principal symbol is $q_{I, \ell}$. Its subprincipal symbol vanishes.

The last step of the proof is the symplectic reduction from $S_{\ell_{1}}^{2} \times \ldots \times S_{\ell_{n}}^{2}$ to $\mathcal{M}_{\ell}$. We will show in part 8 that any invariant Toeplitz operator of a Hamiltonian space $(M, G)$ descends to a Toeplitz operator of the quotient $M / / G$. This result was already proved in [6] for torus action and the proof given there extends directly to the case of a compact Lie group. But it does not give any control on the subprincipal symbol. So we propose another proof and we will show that the principal and subprincipal symbols descend to the principal and subprincipal symbols of the reduced Toeplitz operator. The precise statement is the corollary 8.1, which implies theorem 3.2.

4. On the symplectic geometry of polygon spaces. Let $n \geqslant 3$ and $\left(\ell_{1}, \ldots, \ell_{n}\right)$ be a family of positive numbers, not necessarily integral. We assume that

$$
\ell_{j}<\frac{1}{2}\left(\ell_{1}+\ldots+\ell_{n}\right)
$$

for any $j=1, \ldots, n$. Recall that $S_{\ell}^{2}$ is the sphere of $\mathfrak{s u}(2)^{*}$ with radius $\ell$. It is the coadjoint orbit with symplectic volume $2 \pi \ell$. Consider the symplectic quotient

$$
\mathcal{M}_{\ell}=\left\{\left(x_{1}, \ldots, x_{n}\right) \in S_{\ell_{1}}^{2} \times \ldots \times S_{\ell_{n}}^{2} / x_{1}+\ldots+x_{n}=0\right\} / S U(2) .
$$

Since we do not assume $(10), \mathcal{M}_{\ell}$ may have some singularities. They form a finite set $\Sigma_{\ell}$ consisting of the classes $\left[\left(x_{1}, \ldots, x_{n}\right)\right]$ such that all the $x_{i}$ belong to the same line. $\mathcal{M}_{\ell} \backslash \Sigma_{\ell}$ is a symplectic manifold of dimension $2(n-3)$. It is non-empty because of the inequalities (13).

In section 4.1, we associate to each admissible graph an integrable system of $\mathcal{M}_{\ell}$ and give its properties. The motivation is to obtain next the best description of the joint eigenstates of the operators $\left(H_{a, \ell}\right)$. In section 4.2 , we make clear the relationship with the decomposition of polygons into triangles. We also introduce natural action-angle coordinates of the integrable system. The proofs of the various results are postponed to the next sections.

4.1. An integrable system. Let $\Gamma$ be an admissible graph with $n$ half-edges. Assume that the internal edges are oriented. Then for any internal edge $a$, denote by $I(a)$ the set of half-edges which are connected to the initial vertex of $a$ by a path which doesn't contain the terminal vertex of $a$. Introduce the length function $\lambda_{a}$ of $\mathcal{M}_{\ell}$ defined by

$$
\lambda_{a}\left(\left[x_{1}, \ldots, x_{n}\right]\right)=\left|\sum_{i \in I(a)} x_{i}\right|
$$

and denote by $h_{a}$ the square of $\lambda_{a}$. Observe that $\lambda_{a}$ is defined independently of the direction of $a$. Using this, it is easy to see that the Poisson bracket $\left\{h_{a}, h_{b}\right\}=0$ vanishes for any two internal edges. 
Denote by $\mathcal{M}_{\ell, \Gamma}^{*}$ the subset of $\mathcal{M}_{\ell} \backslash \Sigma_{\ell}$ where none of the functions $\lambda_{a}$ vanishes. The functions $\lambda_{a}$ are smooth on $\mathcal{M}_{\ell, \Gamma}^{*}$ and they mutually Poisson commute. To describe the joint image of the $\lambda_{a}$, we introduce some more notations.

Let us denote by $E(\Gamma)$ the set of edges of $\Gamma$. Let $\Delta(\Gamma)$ be the convex polyhedron of $\mathbb{R}^{E(\Gamma)}$ which consists of the $(2 n-3)$-tuples $\left(d_{a}\right)$ of non-negative real numbers such that for any three mutually distinct edges $a, b$ and $c$ incident to the same vertex, $d_{a}$, $d_{b}$ and $d_{c}$ are the lengths of an Euclidean triangle, that is the inequalities $\left|d_{a}-d_{b}\right| \leqslant$ $d_{c} \leqslant d_{a}+d_{b}$ hold. Let $\Delta(\ell, \Gamma)$ be the convex polyhedron of $\mathbb{R}^{E_{\text {int }}(\Gamma)}$

$$
\Delta(\ell, \Gamma)=\left\{\left(d_{a}\right)_{a \in E_{\mathrm{int}}(\Gamma)} /\left(\ell_{i}, d_{a}\right)_{i \in E_{\mathrm{half}}(\Gamma), a \in E_{\mathrm{int}}(\Gamma)} \in \Delta(\Gamma)\right\} .
$$

The following theorem will be proved in section 4.3.

TheOREM 4.1. Let $n \geqslant 4$ and let $\Gamma$ be an admissible graph with $n$ half-edges. Let $\left(\ell_{i}\right)_{i=1, \ldots, n}$ be a family of positive real numbers satisfying (13). The image of the map

$$
\lambda: \mathcal{M}_{\ell} \rightarrow \mathbb{R}^{E_{\text {int }}(\Gamma)}, \quad x \rightarrow\left(\lambda_{a}(x)\right)
$$

is the polyhedron $\Delta(\ell, \Gamma)$. The fibers of $\lambda$ are connected. Denote by $\operatorname{Int}(\Delta(\ell, \Gamma))$ the interior of $\Delta(\ell, \Gamma)$. Then the set

$$
\mathcal{M}_{\ell, \Gamma}^{\mathrm{reg}}:=\lambda^{-1}(\operatorname{Int} \Delta(\ell, \Gamma))
$$

is open and dense in $\mathcal{M}_{\ell}$ and included in $\mathcal{M}_{\ell, \Gamma}^{*}$. For any $x \in \mathcal{M}_{\ell, \Gamma}^{\mathrm{reg}}$, the differentials $d_{x} \lambda_{a}, a \in E_{\text {int }}(\Gamma)$, are linearly independent.

This result is strongly related to theorem 2.1 about the spectrum of the operators $\left(H_{a}\right)$. The admissible colorings of the graph $\Gamma$ are particular points of the polyhedron $\Delta(\Gamma)$. The relationship between a color $\varphi(a)$ and the eigenvalue $\varphi(a)(\varphi(a)+1)$ is similar to the relationship between $\lambda_{a}$ and its square $h_{a}$. These analogies will be made more precise in part 5.2 where we shall state the Bohr-Sommerfeld conditions. To prepare the description of the joint eigenstates of the $\left(H_{a, \ell}\right)$ as Lagrangian sections, we explain now how the Hamiltonian flow of the $\lambda_{a}$ defines a torus action. The following result is proved in section 4.4

THEOREM 4.2. Under the same assumptions as theorem 4.1, the Hamiltonian flows on $\mathcal{M}_{\ell, \Gamma}^{*}$ of the $\frac{1}{2} \lambda_{a}, a \in E_{\text {int }}(\Gamma)$, mutually commute and are $2 \pi$-periodical. Consequently they define a torus action

$$
\mathbb{T}^{E_{\text {int }}(\Gamma)} \times \mathcal{M}_{\ell, \Gamma}^{*} \rightarrow \mathcal{M}_{\ell, \Gamma}^{*}
$$

This action is free over $\mathcal{M}_{\ell, \Gamma}^{\mathrm{reg}}$. The fibres of $\lambda$ in $\mathcal{M}_{\lambda, \Gamma}^{\mathrm{reg}}$ are the torus orbits.

As we will see in the next section, there exist natural angle functions which form together with the $\frac{1}{2} \lambda_{a}$ an action-angle coordinate system. When the $\ell_{i}$ are integers and satisfy the condition (11), there exist other remarkable action coordinates $\gamma_{a}$ associated to the prequantum bundle $L_{\ell}$. They are defined modulo $\mathbb{Z}$ by the condition that $e^{2 i \pi \gamma_{a}(x)}$ is the holonomy in $L_{\ell}$ of the loop

$$
t \in[0, \pi] \rightarrow \Phi_{a, t}(x)
$$

where $\Phi_{a, t}$ is the Hamiltonian flow of $\lambda_{a}$ at time $t$. We will prove in part 4.5 the following

Proposition 4.1. For any internal edge a, we have $\gamma_{a}=\frac{1}{2}\left(\lambda_{a}+\sum_{i \in I(a)} \ell_{i}\right)$. 


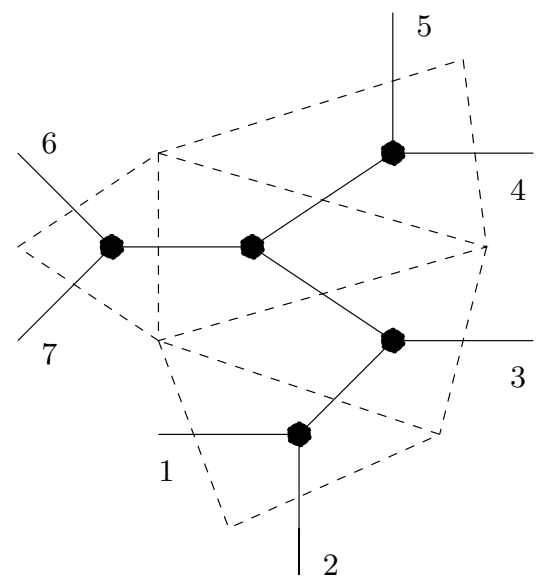

FIG. 6. Triangulation

4.2. Bending flows and action-angle coordinates. Consider first a polygon with $n$ sides numbered from 1 to $n$. Let us cut the polygon into $n-2$ triangles by connecting the vertices with $n-3$ straight lines. To this triangulation is associated a admissible graph $\Gamma$ defined as follows. The half-edges of $\Gamma$ are the sides of the polygon. The internal edges are the straight lines connecting the vertices of the polygon. The vertices of $\Gamma$ are the triangles. The edges incident to a vertex are the sides of the triangle, cf. figure 6 . It is easily seen that this graph is admissible. Furthermore any admissible graph may be obtained in this way.

In the following, we consider a triangulation of a $n$-sided polygon $P$ with its associated admissible graph $\Gamma$. We assume that the sides of the polygon are numbered in the apparent order. Then to each $n$-tuple $x=\left(x_{1}, \ldots, x_{n}\right)$ of $\mathfrak{s u}^{*}(2)$ we associate the polygon $P(x)$ with vertices $0, x_{1}, x_{1}+x_{2}, \ldots, x_{1}+\ldots+x_{n-1}$. So each class $\left[x_{1}, \ldots, x_{n}\right]$ of $\mathcal{M}_{\ell}$ represents a polygons of $\mathfrak{s u}^{*}(2)$ up to isometry. The triangulation of $P$ induces a triangulation of $P\left(x_{1}, \ldots, x_{n}\right)$. Observe that for each internal edge $a$ of $\Gamma, \lambda_{a}(x)$ is the length of a internal edge of the triangulation. This explains that the image of $\lambda$ is the polyhedron $\Delta(\ell, \Gamma)$ as asserted in theorem 4.1. Indeed to each vertex of $\Gamma$ corresponds a face of the triangulation and related triangle inequalities.

In the proof of theorem 4.2, we shall show that the Hamiltonian flow of $\lambda_{a}$ at time $t$ maps $x=\left[x_{1}, \ldots, x_{n}\right] \in \mathcal{M}_{\ell}^{*}$ into $\Phi_{a}(x, t)=\left[z_{1}, \ldots, z_{n}\right]$ where

$$
z_{i}=\left\{\begin{array}{l}
\operatorname{Ad}_{g} x_{i} \text { if } i \in I(a) \\
x_{i} \text { otherwise }
\end{array}, \quad \text { with } g=\exp \left(\frac{t}{\lambda_{a}(x)} \sum_{i \in I(a)} x_{i}\right) .\right.
$$

Here we identified $\mathfrak{s u}(2)$ and $\mathfrak{s u}(2)^{*}$ by using the invariant scalar product introduced above. Considering the previous interpretation of $\mathcal{M}_{\ell}$, these flows are bendings along the internal edges of the triangulation.

Now for any internal edge $a$, introduce a coordinate

$$
\theta_{a}: \mathcal{M}_{\ell, \Gamma}^{\mathrm{reg}} \rightarrow \mathbb{R} / 2 \pi \mathbb{Z}
$$

which measures the dihedral angle between the two faces adjacent to the internal edge of the triangulation associated to $a$. More precisely, we require that $\theta_{a}=0$ or $\pi$ when 
these faces are coplanar and that $\theta_{a}\left(\Phi_{a}(x, t)\right)=\theta_{a}+t$.

TheOREM 4.3. The subspace $\mathcal{P}_{\ell}$ of $\mathcal{M}_{\ell} \backslash \Sigma_{\ell}$ which consists of classes of $n$-tuples of coplanar vectors, is a closed Lagrangian submanifold. Consequently, the family $\frac{1}{2} \lambda_{a}, \theta_{a}, a \in E_{\mathrm{int}}(\Gamma)$, is an action-angle coordinate system, that is the symplectic form is given by

$$
\omega=\sum_{a \in E_{\text {int }}(\Gamma)} d\left(\frac{1}{2} \lambda_{a}\right) \wedge d \theta_{a}
$$

on $\mathcal{M}_{\ell, \Gamma}^{\mathrm{reg}}$.

This theorem was proved in [14] for the graph $\Gamma_{n}$ and its associated triangulation given in figures 4 and 5 .

Proof. It is easily seen that $\mathcal{P}_{\ell}$ is a $(n-3)$-dimensional submanifold of $\mathcal{M}_{\ell} \backslash$ $\Sigma_{\ell}$. Let $R$ be a reflexion of $\mathfrak{s u}^{*}(2)$. Consider the involution $\Psi$ of $\mathcal{M}_{\ell} \backslash \Sigma_{\ell}$ which sends $\left[x_{1}, \ldots, x_{n}\right]$ into $\left[R\left(x_{1}\right), \ldots, R\left(x_{n}\right)\right]$. It transforms the symplectic form into its opposite. $\mathcal{P}_{\ell}$ being the fixed point set of $\Psi$, it is a Lagrangian submanifold.

4.3. Proof of theorem 4.1. Let $H$ be the subspace of $(\mathfrak{s u}(2))^{n}$

$$
H:=\left\{\left(x_{i}\right) \in(\mathfrak{s u}(2))^{n} / x_{1}+\ldots+x_{n}=0\right\} .
$$

Denote by $E(\Gamma)$ the set of edges of $\Gamma$ and introduce the map $\underline{\lambda}: H \rightarrow \mathbb{R}^{E(\Gamma)}$ whose components are

$$
\underline{\lambda}_{a}(x)=\left\{\begin{array}{l}
\left|\sum_{i \in I(a)} x_{i}\right| \quad \text { if } a \text { is an internal edge, } \\
\left|x_{a}\right| \quad \text { if } a \text { is an half-edge. }
\end{array}\right.
$$

Recall that $\Delta(\Gamma)$ is the convex polyhedron of $\mathbb{R}^{E(\Gamma)}$ consisting of the $\left(d_{a}\right)$ such that for any three edges $a, b$, and $c$ mutually distinct and incident to the same vertex, $d_{a}$, $d_{b}$ and $d_{c}$ satisfy the triangle inequalities.

TheOREM 4.4. The image of $\underline{\lambda}$ is the set $\Delta(\Gamma)$. The fibres of $\underline{\lambda}$ are connected. Furthermore the interior points of $\Delta(\Gamma)$ are regular values of $\underline{\lambda}$.

The interior of $\Delta(\Gamma)$ consists of the $\left(d_{a}\right)_{a \in E(\Gamma)}$ such that the strict triangle inequalities

$$
\left|d_{a}-d_{b}\right|<d_{c}<d_{a}+d_{b}
$$

hold at each vertex. So the functions $\underline{\lambda}_{a}$ do not vanish and are smooth on $\underline{\lambda}^{-1}(\operatorname{Int}(\Delta(\Gamma)))$.

Before the proof, let us deduce theorem 4.1 from 4.4. We immediately have that the image of $\lambda$ is the polyhedron $\Delta(\ell, \Gamma)$ and that its fibre are connected. Next it is easily checked that the interior of $\Delta(\ell, \Gamma)$ is the set of $\left(d_{a}\right)_{a \in E_{\text {int }}(\Gamma)}$ such that $\left(d_{a}, \ell_{i}\right)$ belongs to $\operatorname{Int}(\Delta)$. So theorem 4.4 implies that the differentials of the $\lambda_{a}$ are linearly independent over $\lambda^{-1}(\operatorname{Int} \Delta(\ell, \Gamma))$. It remains to show the density of $\lambda^{-1}(\operatorname{Int} \Delta(\ell, \Gamma))$, which follows from proposition 4.2.

Proof of theorem 4.4. The proof is by induction on the number of half-edges. The result is easily proved for the graph with three monovalent vertices. Observe that in this case the fibers of $\underline{\lambda}$ are the orbits of the diagonal $S U(2)$-action. Furthermore 
$\left(x_{1}, x_{2}, x_{3}\right)$ is a regular point of $\underline{h}$ if the triangle with vertices $0, x_{1}, x_{1}+x_{2}$ is nondegenerate.

Let $n \geqslant 3$. Consider an admissible graph $\Gamma$ with $(n+1)$ half-edges. Then $\Gamma$ admits a vertex $\underline{v}$ incident to two half-edges and an internal edge. To see this, consider the graph obtained from $\Gamma$ by deleting all the half-edges. This new graph being a tree, it has at least two monovalent vertices, these vertices satisfy the property. Next we order the half-edges of $\Gamma$ in such a way that the two ones incident to $\underline{v}$ are the $n$-th and $(n+1)$-th. Denote by $\underline{a}$ the internal edge incident to $\underline{v}$. Changing the directions of the internal edges if necessary, we may assume that

$$
I(\underline{a})=\{n, n+1\}, \quad I(a) \subset\{1, \ldots, n-1\} .
$$

Let $\Gamma^{\prime}$ be the graph obtained from $\Gamma$ by removing the vertex $\underline{v}$ and the $n$-th and $(n+1)$-th half-edges. Denote by $\underline{\lambda}^{\prime}$ and $\underline{\lambda}^{\prime \prime}$ the maps associated respectively to $\Gamma^{\prime}$ and the graph $\Gamma^{\prime \prime}$ with three monovalent vertices. Then the induction is based on the simple observation that $\underline{\lambda}(x)=d$ if and only if

$$
\underline{\lambda}^{\prime}\left(x_{1}, \ldots, x_{n-1},-y\right)=\left.d\right|_{E\left(\Gamma^{\prime}\right)} \quad \text { and } \quad \underline{\lambda}^{\prime \prime}\left(y, x_{n}, x_{n+1}\right)=\left(|y|, d_{n}, d_{n+1}\right)
$$

where $y=x_{1}+\ldots+x_{n-1}$. As a first consequence, we immediately deduce that the image of $\lambda$ is $\Delta(\Gamma)$ if we already know that the images of $\lambda^{\prime}$ and $\lambda^{\prime \prime}$ are the polyhedra $\Delta\left(\Gamma^{\prime}\right)$ and $\Delta\left(\Gamma^{\prime \prime}\right)$.

Let us prove that the fibers of $\underline{\lambda}$ are connected. Let $x^{0}$ and $x^{1}$ be such that $\underline{\lambda}\left(x^{0}\right)=\underline{\lambda}\left(x^{1}\right)$. Denote by $x^{\prime}$ the $(n-1)$-tuple $\left(x_{1}, \ldots x_{n-1}\right)$ and by $y$ the sum $x_{1}+\ldots+x_{n-1}$. Assuming the fibers of $\underline{\lambda}^{\prime}$ are connected, there exists a path

$$
t \rightarrow\left(x^{\prime t},-y^{t}\right)
$$

from $\left(x^{\prime 0},-y^{0}\right)$ to $\left(x^{\prime 1},-y^{1}\right)$ remaining in the same fiber of $\underline{\lambda}^{\prime}$. Since $\left|y^{t}\right|$ is constant, there exists a path $t \rightarrow g^{t}$ in $S U(2)$ such that $g^{t} y^{0}=y^{t}$. Then the path

$$
t \rightarrow\left(x^{\prime t}, g^{t} x_{n}^{0}, g^{t} x_{n+1}^{0}\right)
$$

connects $x^{0}$ with $\left(x^{\prime 1}, g^{1} x_{n}^{0}, g^{1} x_{n+1}^{0}\right)$ and takes its value in a single fiber of $\underline{\lambda}$. Next since

$$
\underline{\lambda}^{\prime \prime}\left(-g^{1}\left(x_{n}^{0}+x_{n+1}^{0}\right), g^{1} x_{n}^{0}, g^{1} x_{n+1}^{0}\right)=\underline{\lambda}^{\prime \prime}\left(-\left(x_{n}^{1}+x_{n+1}^{1}\right), x_{n}^{1}, x_{n+1}^{1}\right),
$$

there exists a path

$$
t \rightarrow\left(-\left(z_{n}^{t}+z_{n+1}^{t}\right), z_{n}^{t}, z_{n+1}^{t}\right)
$$

which connects these two points by remaining in the same fiber of $\underline{\lambda}^{\prime \prime}$. Furthermore since $g^{1}\left(x_{n}^{0}+x_{n+1}^{0}\right)=y^{1}=x_{n}^{1}+x_{n+1}^{1}$, one may choose this path in such a way that $\left(z_{n}^{t}+z_{n+1}^{t}\right)$ is constant. Finally the path

$$
t \rightarrow\left(x^{1}, z_{n}^{t}, z_{n+1}^{t}\right)
$$

connects $\left(x^{\prime 1}, g^{1} x_{n}^{0}, g^{1} x_{n+1}^{0}\right)$ with $x^{1}$ and this ends the proof of the connectedness.

Let us prove that the interior points of $\Delta(\Gamma)$ are regular values of $\underline{\lambda}$. Assume that the result is satisfied for the function $\underline{\lambda}^{\prime}$ associated to $\Gamma^{\prime}$. Let $x^{0}$ be such that its image belongs to the interior of $\Delta(\Gamma)$. Then one has to prove that the map

$$
(\mathfrak{s u}(2))^{n+1} \rightarrow \mathbb{R}^{E(\Gamma)} \times \mathfrak{s u}(2), \quad x \rightarrow\left(\underline{\lambda}(x), x_{1}+\ldots+x_{n+1}\right)
$$


is submersive at $x^{0}$, where $\underline{\lambda}$ is the obvious extension of $\underline{\lambda}$ from $H$ to $(\mathfrak{s u}(2))^{n+1}$. Consider the isomorphism

$$
\Psi:(\mathfrak{s u}(2))^{n+1} \rightarrow H^{\prime} \times H^{\prime \prime}, \quad x \rightarrow\left(x^{\prime},-y\right),\left(-\left(x_{n}+x_{n+1}\right), x_{n}, x_{n+1}\right) .
$$

Here we denote as previously by $x^{\prime}$ the $(n-1)$-tuple $\left(x_{1}, \ldots x_{n-1}\right)$ and by $y$ the sum $x_{1}+\ldots+x_{n-1}$. Then one has

$$
\underline{\lambda}_{a}^{\prime} \circ \Psi=\underline{\lambda}_{a}, \quad \forall a \in E\left(\Gamma^{\prime}\right) \quad \text { and } \quad \underline{\lambda}^{\prime \prime} \circ \Psi=\left(\underline{\lambda}_{a}, \underline{\lambda}_{n}, \underline{\lambda}_{n+1}\right) .
$$

By induction assumption, $\underline{\lambda}^{\prime}$ is submersive at $\left(x^{\prime 0},-y^{0}\right)$. Hence it suffices to prove that the map

$$
H^{\prime \prime} \rightarrow \mathbb{R}^{2} \times \mathfrak{s u}(2), \quad\left(-\left(x_{n}+x_{n+1}\right), x_{n}, x_{n+1}\right) \rightarrow\left(\left|x_{n}\right|,\left|x_{n+1}\right|, x_{n}+x_{n+1}\right)
$$

is submersive at $\left(-\left(x_{n}^{0}+x_{n+1}^{0}\right), x_{n}^{0}, x_{n+1}^{0}\right)$. This is true because $\underline{\lambda}\left(x^{0}\right)$ being an interior point of $\Delta(\Gamma)$, the triangle with vertices $0, x_{n}^{0}$ and $x_{n}^{0}+x_{n+1}^{0}$ is non-degenerate. This ends the proof of the theorem. $\mathrm{Q}$

For any $n$-tuple $\ell$ of positive numbers, denote by $P_{\ell}$ the subset of $H$,

$$
P_{\ell}:=\left(S_{\ell_{1}}^{2} \times \ldots \times S_{\ell_{n}}^{2}\right) \cap H,
$$

so that $\mathcal{M}_{\ell}$ is the quotient of $P_{\ell}$ by $S U(2)$.

Proposition 4.2. If $\ell$ satisfies (13), then $\underline{\lambda}^{-1}(\Delta(\Gamma)) \cap P_{\ell}$ is dense in $P_{\ell}$.

Proof. Again the proof is by induction on the number of half-edges. Introduce the graphs $\Gamma, \Gamma^{\prime}$ and $\Gamma^{\prime \prime}$ as in the proof of theorem 4.4 and assume the result is satisfied for $\Gamma^{\prime}$ and for any $\ell$ satisfying the inequalities (13).

Let $\ell$ be a $(n+1)$-tuple satisfying (13). Let $\tilde{\Sigma}_{\ell}$ be the set of $\left(x_{1}, \ldots, x_{n+1}\right) \in P_{\ell}$ such that the $x_{i}$ are mutually colinear. It is easily deduced from (13) that $P_{\ell} \backslash \tilde{\Sigma}_{\ell}$ is dense in $P_{\ell}$.

Next we consider the set $Q$ consisting of the $\left(x_{1}, \ldots, x_{n+1}\right) \in P_{\ell} \backslash \tilde{\Sigma}_{\ell}$ such that the lengths $\ell_{n}^{\prime}=\left|x_{n}+x_{n+1}\right|$ satisfy the two following conditions. First $\left(\ell_{1}, \ldots, \ell_{n-1}, \ell_{n}^{\prime}\right)$ satisfies the inequalities (13) and second $\ell_{n}^{\prime}, \ell_{n}, \ell_{n+1}$ satisfy the strict triangle inequalities. One proves that $Q$ is dense in $P_{\ell} \backslash \Sigma_{\ell}$. To do this observe that the inequalities (13) are satisfied as soon as there is no equality and this can happen only for a finite number of $\ell_{n}^{\prime}$. For any $x^{0} \in P_{\ell} \backslash \tilde{\Sigma}_{\ell}$, one has to find points in $Q$ arbitrarily close to $x^{0}$. This can be proved by considering separetly the case where $x_{1}^{0}, \ldots, x_{n-1}^{0}$ are mutually colinear. If there are not, one concludes by using that the map

$$
S_{\ell_{1}} \times \ldots \times S_{\ell_{n-1}} \rightarrow \mathfrak{s u}(2)^{*}
$$

if submersive at $\left(x_{1}^{0}, \ldots, x_{n-1}^{0}\right)$.

The last step is to prove the density of $\underline{\lambda}^{-1}(\Delta(\Gamma)) \cap P_{\ell}$ in $Q$. This follows from the induction assumption.

4.4. Proof of theorem 4.2. The Hamiltonian flow of the $\lambda_{a}$ is easily described applying the following general result. Let $(M, \omega)$ be a symplectic manifold with a Hamiltonian action of a Lie group $G$. Assume that the Lie algebra $\mathfrak{g}$ has an invariant scalar product, which we use to identify $\mathfrak{g}$ and $\mathfrak{g}^{*}$. Denote by $\mu$ the momentum of 
the action and let $M^{*}$ be the open set $\{\mu \neq 0\}$ of $M$. One easily checks the following proposition.

Proposition 4.3. The Hamiltonian flow of $|\mu| \in \mathcal{C}^{\infty}\left(M^{*}\right)$ at time $t$ is given by

$$
\Phi_{t}(x)=\exp \left(t \frac{\mu(x)}{|\mu(x)|}\right) \cdot x, \quad \forall x \in M^{*} .
$$

Let $I$ be a subset of $\{1, \ldots, n\}$. Consider the Hamiltonian action of $S U(2)$ on $S_{\ell_{1}}^{2} \times \ldots \times S_{\ell_{n}}^{2}$ with momentum

$$
\mu_{I}\left(x_{1}, \ldots, x_{n}\right)=\sum_{i \in I} x_{i}
$$

By the previous proposition, the flow $\rho_{I, t}$ of $\left|\mu_{I}\right|$ at time $t$ sends $\left(x_{1}, \ldots, x_{n}\right)$ into the $n$-tuple $\left(y_{1}, \ldots, y_{n}\right)$ given by

$$
y_{i}=\left\{\begin{array}{l}
\operatorname{Ad}_{g} x_{i} \text { if } i \in I, \\
x_{i} \text { otherwise, }
\end{array} \quad \text { with } g=\exp \left(t \frac{\mu_{I}(x)}{\left|\mu_{I}(x)\right|}\right) .\right.
$$

We apply this to the set $I(a)$ associated to an internal edge $a$. The function $\left|\mu_{I(a)}\right|$ is invariant by the diagonal action and descends to the function $\lambda_{a}$. Hence the flows $\rho_{I(a), t}$ lifts the Hamiltonian flow of $\lambda_{a}$. Furthermore, with our normalization,

$$
\exp (\xi)=1 \Leftrightarrow|\xi| \in 2 \pi \mathbb{N}, \quad \forall \xi \in \mathfrak{s u}(2) .
$$

Since the coadjoint action factorizes through a $S O(3)$-action, the flow of the $\lambda_{a}$ is $\pi$-periodical and this proves the first part of theorem 4.2. Next by theorem 4.1, the torus orbits in $\mathcal{M}_{\ell, \Gamma}^{\mathrm{reg}}$ and the fibers of $\lambda$ have the same dimension. Furthermore the fibers are connected. Hence the fibers in $\mathcal{M}_{\ell, \Gamma}^{\text {reg }}$ are the torus orbits. It remains to prove that the torus action is free in $\mathcal{M}_{\ell, \Gamma}^{\mathrm{reg}}$.

Let $H$ be the subspace defined in (14). Let us extend the action $\rho_{I, t}$ on $H \backslash$ $\left\{\sum_{i \in I} x_{i} \neq 0\right\}$ in the obvious way. We will consider the actions $\rho_{I(a), t}$ altogether. Here we can not choose the orientations of the internal edges in such a way that the sets $I(a)$ are mutually disjoint. So the action of $\rho_{I(a), t}$ and $\rho_{I(b), t}$ do not necessarily commute. Let $a_{1}, \ldots, a_{n-3}$ be the internal edges of $\Gamma$. The following proposition completes the proof of theorem 4.2.

Proposition 4.4. For any $x \in H$ such that $\underline{\lambda}(x) \in \operatorname{Int} \Delta(\Gamma)$ and for any $\left(t_{i}\right) \in$ $\mathbb{R}^{n-3}$, if there exists $g \in S U(2)$ such that

$$
\rho_{I\left(a_{1}\right), t_{1}}\left(\rho_{I\left(a_{2}\right), t_{2}}\left(\ldots\left(\rho_{I\left(a_{n-3}\right), t_{n-3}}(x)\right) \ldots\right)\right)=g \cdot x
$$

then $t_{1} \equiv \ldots \equiv t_{n} \equiv 0$ modulo $\pi$.

Proof. Again the proof is by induction on the number of half-edges. Introduce the graphs $\Gamma, \Gamma^{\prime}$ and $\Gamma^{\prime \prime}$ as in the proof of theorem 4.4 and assume the result is satisfied for $\Gamma^{\prime}$. Since the various circle actions mutually commute modulo the diagonal action of $S U(2)$, the result is independent of the order of the internal edges. Observe also that

$$
\rho_{I, t}(x)=g(x, t) \cdot \rho_{I^{c}, t}(x) .
$$


So the result does not depend on the direction of the internal edge. So we may assume that $a_{n-2}$ is the internal edge of $\Gamma$ incident to the $n$-th and $(n+1)$-th halfedges. We may also assume that $I\left(a_{n-2}\right)=\{n, n+1\}$ and $I\left(a_{k}\right) \subset\{1, \ldots, n-1\}$ for $k=1, \ldots, n-3$. Let $x \in \underline{\lambda}^{-1}(\operatorname{Int} \Delta(\Gamma))$ such that

$$
\rho_{I\left(a_{1}\right), t_{1}}\left(\rho_{I\left(a_{2}\right), t_{2}}\left(\ldots\left(\rho_{I\left(a_{n-2}\right), t_{n-2}}(x)\right) \ldots\right)\right)=g \cdot x .
$$

This implies that

$$
\rho_{I\left(a_{1}\right), t_{1}}\left(\rho_{I\left(a_{2}\right), t_{2}}\left(\ldots\left(\rho_{I\left(a_{n-3}\right), t_{n-3}}\left(x^{\prime},-y\right)\right) \ldots\right)\right)=g \cdot\left(x^{\prime},-y\right)
$$

where $x^{\prime}=\left(x_{1}, \ldots, x_{n-1}\right)$ and $y=x_{1}+\ldots+x_{n-1}$. Furthermore

$$
\exp \left(t_{n-2} \frac{x_{n}+x_{n+1}}{\left|x_{n}+x_{n+1}\right|}\right) \cdot\left(x_{n}, x_{n+1}\right)=g \cdot\left(x_{n}, x_{n+1}\right) .
$$

By the induction assumption, one has that $t_{1} \equiv \ldots \equiv t_{n-3} \equiv 0$ modulo $\pi$. Consequently, (17) reads as

$$
\left(x_{1}, \ldots, x_{n-1}\right)=g \cdot\left(x_{1}, \ldots, x_{n-1}\right)
$$

If $g \neq \pm \mathrm{id}$, this implies that the vectors $x_{1}, \ldots, x_{n-1}$ are colinear and this contradicts the fact that $\underline{\lambda}(x) \in \operatorname{Int}(\Delta(\Gamma))$. So $g= \pm$ id. Finally since $x_{n}$ and $x_{n+1}$ are not colinear, equation (18) implies that $t_{n-2} \equiv 0$ modulo $\pi$.

4.5. Computation of the holonomies. Extending the proposition 4.3 to the prequantum case, we can also compute the actions $\gamma_{a}$. Assume that $(M, \omega)$ admits a prequantization bundle $L \rightarrow M$ with curvature $\frac{1}{i} \omega$ and that the action of $G$ lifts to $L$ satisfying the usual assumption (6).

Proposition 4.5. For any $x \in M^{*}$ and $u \in L_{x}$, one has

$$
e^{-i t|\mu(x)|} \mathcal{T}_{t} \cdot u=\exp \left(t \frac{\mu(x)}{|\mu(x)|}\right) \cdot u
$$

where $\mathcal{T}_{t} . u \in L_{\Phi_{t}(x)}$ is the parallel transport of $u$ along the path $s \in[0, t] \rightarrow \Phi_{s}(x)$.

To deduce proposition 4.1, we apply this result at time $\pi$ to the Hamiltonian action with momentum $\mu_{I(a), t}$ introduced in (16). Be careful that the action on the prequantization bundle factorizes through a $S O(3)$-action only if $\sum_{i \in I(a)} \ell_{i}$ is even.

5. Semi-classical properties of the joint eigenstates. Let $\Gamma$ be an admissible graph with $n$ half-edges and let $\left(\ell_{i}\right)_{i=1, \ldots, n}$ be a family of positive integers. For any integer $k \geqslant 1$, we defined in section 2.4 a family

$$
\left\{H_{a, k \ell-1} ; a \in E_{\text {int }}(\Gamma)\right\}
$$

of mutually commuting operators of $\mathcal{H}_{k \ell-1}$. For any joint eigenvalue $E=\left(E_{a}\right) \in$ $\mathbb{R}^{E_{\text {int }}(\Gamma)}$ of the operators $k^{-2} H_{a, k \ell-1}$, introduce a unitary eigenvector $\Psi_{E, k}$

$$
\frac{1}{k^{2}} H_{a, k \ell-1} \Psi_{E, k}=E_{a} \Psi_{E, k}, \quad \forall a \in E_{\text {int }}(\Gamma) .
$$

Assume furthermore that $\ell$ satisfies assumptions (9), (10) and (11). Then by theorem $3.1, \mathcal{H}_{k \ell-1}$ is isomorphic to $H^{0}\left(\mathcal{M}_{\ell}, L_{\ell}^{k} \otimes \delta_{\ell}\right)$ and by theorem 3.2 the sequence

$$
T_{a}=\left(\frac{1}{k^{2}} V_{k} H_{a, k \ell-1} V_{k}^{-1}\right)_{k=1,2, \ldots}
$$


is a Toeplitz operator with principal symbol

$$
h_{a}=\left|\sum_{i \in I(a)} x_{i}\right|^{2} .
$$

As a consequence we can describe precisely the eigenstates $\Psi_{E, k}$ in the semi-classical limit. This is done in section 5.1. Section 5.2 is devoted to the Bohr-Sommerfeld conditions.

5.1. Eigenstates of the $H_{a}$. First the eigenstates are microlocalised on the level sets of the joint principal symbol $h:=\left(h_{a}\right): \mathcal{M}_{\ell} \rightarrow \mathbb{R}^{E_{\text {int }}(\Gamma)}$. Introduce the closed subset

$$
\Lambda:=\{(x, E) / h(x)=E\}
$$

of $\mathcal{M}_{\ell} \times \mathbb{R}^{E_{\text {int }}(\Gamma)}$

ThEOREM 5.1. For any $(x, E) \notin \Lambda$, there exist neighborhoods $U$ of $x$ in $\mathcal{M}_{\ell}$ and $V$ of $E$ in $\mathbb{R}^{E_{\mathrm{int}}(\Gamma)}$ and a sequence $\left(C_{N}\right)$ of positive real numbers such that

$$
\left|V_{k} \Psi_{E, k}(y)\right| \leqslant C_{N} k^{-N}
$$

for any integers $k$ and $N$, any $y \in U$ and any joint eigenvalue $E \in V$. The same result holds if we replace $V_{k}$ by $V_{k}\left(V_{k}^{*} V_{k}\right)^{-\frac{1}{2}}$.

Next since the family $\left(h_{a}\right)$ is an integrable system as stated in theorem 4.1 , we can determine modulo $O\left(k^{-\infty}\right)$ the eigenvectors $\Psi_{E, k}$ on a neighborhood of the the level set $h^{-1}(E)$ when $E$ is regular value of $h$.

Consider as in theorem 4.1 the regular set $\mathcal{M}_{\ell, \Gamma}^{\mathrm{reg}}=\lambda^{-1}(\operatorname{Int} \Delta(\ell, \Gamma))$. To describe uniformly the various eigenstates, introduce the submanifold

$$
\Lambda^{\mathrm{reg}}:=\Lambda \cap\left(\mathcal{M}_{\ell, \Gamma}^{\mathrm{reg}} \times \mathbb{R}^{E_{\mathrm{int}}(\Gamma)}\right)
$$

of $\mathcal{M}_{\ell} \times \mathbb{R}^{E_{\text {int }}(\Gamma)}$. Denote by $L$ and $\delta$ the pull-backs of the bundles $L_{\ell}$ and $\delta_{\ell}$ by the projection $\mathcal{M}_{\ell} \times \mathbb{R}^{E_{\text {int }}(\Gamma)} \rightarrow \mathcal{M}_{\ell}$.

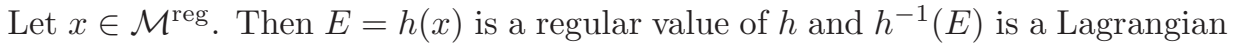
torus described in theorem 4.2. Let us introduce the isomorphism

$$
\varphi_{x}: \delta_{x}^{2} \rightarrow \wedge^{\mathrm{top}, 0}\left(T_{x}^{*} \mathcal{M}\right) \rightarrow \wedge^{\mathrm{top}}\left(T_{x} h^{-1}(E)\right) \otimes \mathbb{C}
$$

Here the first arrow is the isomorphism making $\delta$ a square root of the canonical bundle and the second arrow is the restriction from $T_{x} \mathcal{M}$ to $T_{x} h^{-1}(E)$. Finally denote by $\mu_{E}$ the volume element of $h^{-1}(E)$ which is invariant by the torus action generated by the $\lambda_{a}$ and normalised by

$$
\int_{h^{-1}(E)} \mu_{E}=1
$$

Since there is no prefered orientation of $h^{-1}(E), \mu_{E}$ is uniquely defined up to a sign.

TheOREM 5.2. For any $(x, E) \in \Lambda^{\mathrm{reg}}$, there exist neighborhoods $U$ of $x$ in $\mathcal{M}_{\ell, \Gamma}^{\mathrm{reg}}$ and $V$ of $E$ in $\mathbb{R}^{E_{\mathrm{int}}(\Gamma)}$, a section $F$ of $L \rightarrow U \times V$, a sequence $(g(\cdot, k))_{k}$ of sections 
of $\delta \rightarrow U \times V$, a sequence $\left(D_{k}\right)$ of complex numbers and a sequence $\left(C_{N}\right)$ of positive numbers such that for any integer $k$, any integer $N$, any $y \in U$ and any joint eigenvalue $E \in V$,

$$
\left|V_{k} \Psi_{E, k}(y)-D_{k}\left(\frac{k}{2 \pi}\right)^{\frac{\operatorname{dim} \mathcal{M}}{8}} F^{k}(y, E) g(y, E, k)\right| \leqslant C_{N} k^{-N} .
$$

\section{Furthermore}

1. $\left|D_{k}\right|=1+O\left(k^{-\infty}\right)$

2. For any $E$, the restriction of $F$ to $h^{-1}(E) \times\{E\}$ is flat with a constant unitary norm, $\bar{\partial} F$ vanishes to any order along $\Lambda^{\text {reg }}$ and for all $x \notin \Lambda,|F(x)|<1$.

3. the sequence $(g(\cdot, k))_{k}$ admits an asymptotic expansion

$$
g(y, E, k)=g_{0}(y, E)+k^{-1} g_{1}(y, E)+k^{-2} g_{2}(y, E)+\ldots
$$

for the $\mathcal{C}^{\infty}$ topology, where each coefficient $g_{i} \in \mathcal{C}^{\infty}(U \times V, \delta)$ is such that $\bar{\partial} g_{i}$ vanishes to any order along $\Lambda^{\mathrm{reg}}$.

4. the restriction of the first coefficient $g_{0}$ to $\Lambda^{\mathrm{reg}}$ is such that

$$
\varphi_{y}\left(\left(g_{0}(x, E)\right)^{\otimes 2}\right)=\mu_{E}(y), \quad \forall(y, E) \in \Lambda^{\text {reg }}
$$

where $\varphi_{y}$ and $\mu_{E}$ are defined in (19) and (20).

The same result holds if we replace $V_{k}$ by $V_{k}\left(V_{k}^{*} V_{k}\right)^{-\frac{1}{2}}$.

This follows from section 3 of [4] and section 3 of [5]. In the following remarks, we discuss the various components of the result and how it should be modified in the case where the subprincipal symbols do not vanish.

REMARK 5.1. The estimations (21) are controlled by the restrictions of $F$ and the $g_{i}$ to $\Lambda^{\text {reg. }}$. More precisely consider a section $\tilde{F}$ of $L \rightarrow U \times V$ and a sequence $(\tilde{g}(\cdot, k))$ of sections of $\delta \rightarrow U \times V$ which satisfy conditions 2. and 3. of theorem 5.2, then the equalities

$$
\left.F\right|_{\Lambda^{\mathrm{reg}}}=\left.\tilde{F}\right|_{\Lambda^{\mathrm{reg}}} \quad \text { and }\left.\quad g_{i}\right|_{\Lambda^{\mathrm{reg}}}=\left.\tilde{g}_{i}\right|_{\Lambda^{\mathrm{reg}}} \quad \text { for } \quad i=1, \ldots, N
$$

imply that

$$
\tilde{F}^{k}(x, E) \tilde{g}(x, E, k)=F^{k}(x, E) g(x, E, k)+O\left(k^{-N-1}\right)
$$

uniformly on any compact set of $U \times V$.

REMARK 5.2. The restriction of $F$ to $\Lambda^{\text {reg }}$ is determined up to a factor $f(E)$ by the condition that it is flat along $h^{-1}(E) \times\{E\}$ for any $E$. Equivalently, this condition may be written as

$$
\nabla_{X_{a}}^{L} F=0 \quad \text { on } \Lambda^{\mathrm{reg}}, \quad \forall a \in E_{\mathrm{int}}(\Gamma)
$$

where $X_{a}$ is the Hamiltonian vector field of $h_{a}$. In a similar way the function $g_{0}$ satisfies the transport equations

$$
\mathcal{L}_{X_{a}}^{\delta} g_{0}=0 \quad \text { on } \Lambda^{\text {reg }}, \quad \forall a \in E_{\text {int }}(\Gamma) .
$$

Furthermore the sections $F$ and $g_{0}$ are normalized in condition 2. and 4. in such a way that $\left|D_{k}\right|=1+O\left(k^{-1}\right)$. This follows from theorem 3.2 of [5] and condition (20). 
REMARK 5.3. If the subprincipal symbols $h_{a}^{1}$ of the $T_{a}$ had not vanished, we should replace the transport equations (22) by

$$
\mathcal{L}_{X_{a}}^{\delta} g_{0}+i h_{a}^{1} g_{0}=0 \quad \text { on } \Lambda^{\mathrm{reg}}, \quad \forall a \in E_{\mathrm{int}}(\Gamma) .
$$

5.2. Bohr-Sommerfeld conditions. Bohr-Sommerfeld conditions are the conditions to patch together the sections

$$
F^{k}(\cdot, E) g_{0}(\cdot, E)
$$

of theorem 5.2 modulo an error $O\left(k^{\frac{\operatorname{dim} \mathcal{M}}{8}-1}\right)$. By remarks 5.1 and 5.2, this can be achieved if and only if the restriction to $h^{-1}(E)$ of the bundle $L_{\ell}^{k} \otimes \delta_{\ell}$ is trivial as a flat bundle. Here we consider the flat structure of

$$
\delta_{\ell} \rightarrow h^{-1}(E)
$$

defined in such a way that the local sections $g_{0}(\cdot, E)$ satisfying condition 4 . of theorem 5.2 are flat. The flat structure of

$$
L_{\ell}^{k} \rightarrow h^{-1}(E)
$$

is the one induced by the connection of the prequantum bundle. It is flat because the curvature of $L$ is the symplectic form and $h^{-1}(E)$ is Lagrangian.

For any internal edge $a$ of $\Gamma$, consider an integral curve of the hamiltonian flow of $h_{a}$ in $h^{-1}(E)$. The family of these curves is a base of the first group of homology of $h^{-1}(E)$. One deduces the holonomy of these curves from proposition 4.1. We obtain the Bohr-Sommerfeld conditions.

Proposition 5.1. The restriction to $h^{-1}(E)$ of the bundle $L_{\ell}^{k} \otimes \delta_{\ell}$ is a trivial flat bundle if and only if

$$
\frac{k}{2}\left(E_{a}^{\frac{1}{2}}+\sum_{i \in I(a)} \ell_{i}\right)+\frac{1}{2} \epsilon_{a}(E) \in \mathbb{Z}, \quad \forall a \in E_{\text {int }}(\Gamma)
$$

where $\epsilon_{a}(E)$ is equal to 0 or 1 according to whether the restriction of $\delta_{\ell}$ to the integral curve of $h_{a}$ is trivial or not.

The indices $\epsilon_{a}(E)$ replace the Maslov indices for the usual Bohr-Sommerfeld conditions. It is proved in section 3 of [4] (cf. also section 3 of [5]) that for any couple $(E, k)$ which satisfies the Bohr-Sommerfeld condition, there exists a joint eigenvalue $\tilde{E}(E, k)$ of the operators $T_{a}$ such that

$$
\tilde{E}(E, k)=E+O\left(k^{-2}\right) .
$$

Let us compare this with the description of the spectrum of the $H_{a, k \ell-1}$ given in theorem 2.1. The joint eigenvalues are indexed by the admissible colorings $\varphi$ of $\Gamma$ such that $\varphi(i)=k \ell_{i}-1$ for any half-edge $i$. Observe that the parity conditions satisfied at each vertex are alltogether equivalent to

$$
\varphi(1)+\varphi(2)+\ldots+\varphi(n) \in 2 \mathbb{Z}
$$


and

$$
\varphi(a)+\sum_{i \in I(a)} \varphi(i) \in 2 \mathbb{Z}
$$

for any internal edge $a$ of $\Gamma$. Here the equation (24) is satisfied because $\varphi(i)=k \ell_{i}-1$ and we assumed (11). Comparing the Bohr-Sommerfeld conditions (23) with the equations (25), we obtain the following

Proposition 5.2. $\epsilon_{a}=\# I(a)+1 \bmod 2 \mathbb{Z}$.

Furthermore, if $(E, k)$ satisfies the Bohr-Sommerfeld condition, then

$$
\tilde{E}(E, k)=E-k^{-2}
$$

and $\tilde{E}(E, k)$ is the joint eigenvalue associated to the coloring $\varphi$ of $\Gamma$ defined by $\varphi(a)=$ $k \lambda_{a}-1$.

6. Scalar product of Lagrangian sections. In this part we compute the asymptotics of the scalar product of two Lagrangian sections. The first section is devoted to algebraic preliminaries.

6.1. A half-form pairing. Consider a symplectic vector space $\left(E^{2 n}, \omega\right)$ with a compatible positive complex structure $J$. Given two transversal Lagrangian subspaces $\Gamma_{1}$ and $\Gamma_{2}$, one has a sesquilinear non-degenerate pairing

$$
\left(\wedge^{n} \Gamma_{1}^{*} \otimes \mathbb{C}\right) \times\left(\wedge^{n} \Gamma_{2}^{*} \otimes \mathbb{C}\right) \rightarrow \mathbb{C}, \quad \alpha, \beta \rightarrow i^{n(2-n)}\left(\pi_{1}^{*} \alpha \wedge \pi_{2}^{*} \bar{\beta}\right) / \omega^{n}
$$

where $\pi_{1}$ (resp. $\pi_{2}$ ) is the projection from $E$ onto $\Gamma_{1}$ (resp. $\Gamma_{2}$ ) with kernel $\Gamma_{2}$ (resp. $\left.\Gamma_{1}\right)$. Next for $i=1$ or 2 , the restriction from $E$ to $\Gamma_{i}$ is an isomorphism

$$
\wedge^{n, 0} E^{*} \rightarrow \wedge^{n} \Gamma_{i}^{*} \otimes \mathbb{C}
$$

Composing these maps with the previous pairing, we obtain a non-degenerate sesquilinear pairing

$$
(\cdot, \cdot)_{\Gamma_{1}, \Gamma_{2}}: \wedge^{n, 0} E^{*} \times \wedge^{n, 0} E^{*} \rightarrow \mathbb{C} .
$$

We will consider a square root of this pairing. To remove the sign ambiguity, let us compare it with the usual Hermitian product of the line $\wedge^{n, 0} E^{*}$

$$
(\alpha, \beta)=i^{n(2-n)} \alpha \wedge \bar{\beta} / \omega^{n} .
$$

Recall that for any endomorphism $A$ of $J \Gamma_{1}$ which is symmetric with respect to the scalar product $\omega(X, J Y)$, the graph of the map $J A: J \Gamma_{1} \rightarrow \Gamma_{1}$ is a Lagrangian subspace of $E=J \Gamma_{1} \oplus \Gamma_{1}$. This defines a bijective correspondence between the set of Lagrangian subspaces transversal to $\Gamma_{1}$ and the affine space of symmetric endomorphisms of $J \Gamma_{1}$. One checks the following lemma with a straightforward computation.

Lemma 6.1. Let $A: J \Gamma_{1} \rightarrow J \Gamma_{1}$ be the endomorphism associated to $\Gamma_{2}$. Then

$$
(\alpha, \beta)_{\Gamma_{1}, \Gamma_{2}}=2^{-n} \operatorname{det}(1-i A)(\alpha, \beta)
$$

for any $\alpha, \beta \in \wedge^{n, 0} E^{*}$. 
In particular the pairing induced by the couple $\left(\Gamma_{1}, J \Gamma_{1}\right)$ is the same as the Hermitian product up to a factor $2^{-n}$.

Let us consider a one-dimensional Hermitian line $\delta$ with an isomorphism $\varphi$ : $\delta^{\otimes 2} \rightarrow \wedge^{n, 0} E^{*}$. Then one defines the sesquilinear pairing of $\delta$ by

$$
(\alpha, \beta)_{\Gamma_{1}, \Gamma_{2}}=\sqrt{\left(\varphi\left(\alpha^{2}\right), \varphi\left(\beta^{2}\right)\right)_{\Gamma_{1}, \Gamma_{2}}}, \quad \forall \alpha \in \delta, \forall \beta \in \delta
$$

The square root is determined in such a way that the pairing depends continuously on $\Gamma_{1}$ and $\Gamma_{2}$ and coincides with the scalar product up to a factor $2^{-n / 2}$ when $\Gamma_{2}=J \Gamma_{1}$. Since the set of Lagrangian transversal to $\Gamma_{1}$ is affine, the square root is well-defined.

6.2. Asymptotics of scalar product of Lagrangian section. Let $\left(M^{2 n}, \omega\right)$ be a Kähler manifold with a prequantization bundle $L$ and a half-form bundle $\delta$. Let $\Gamma_{1}$ and $\Gamma_{2}$ be two Lagrangian submanifolds. For $i=1$ or 2 , consider a sequence

$$
F_{i}^{k} a_{i}(\cdot, k) \in \mathcal{C}^{\infty}\left(M, L^{k} \otimes \delta\right), \quad k=1,2, \ldots
$$

where

- $F_{i}$ is a section of $L$ such that its restriction at $\Gamma_{i}$ is flat of norm $1, \bar{\partial} F_{i}$ vanishes at infinite order along $\Gamma_{i}$ and $\left|F_{i}(x)\right|<1$ if $x \notin \Gamma_{i}$.

- $a_{i}(\cdot, k)$ is a sequence of $\mathcal{C}^{\infty}(M, \delta)$ which admits full asymptotic expansion for the $\mathcal{C}^{\infty}$ topology

$$
a_{i}(\cdot, k)=a_{i, 0}+k^{-1} a_{i, 1}+k^{-2} a_{i, 2}+\ldots
$$

with coefficients $a_{i, \ell}$ in $\mathcal{C}^{\infty}(M, \delta)$.

Recall that the scalar product of two sections of $L^{k} \otimes \delta$ is defined by

$$
\left(\Psi_{1}, \Psi_{2}\right)=\int_{M}\left(\Psi_{1}(x), \Psi_{2}(x)\right)_{L_{x}^{k} \otimes \delta_{x}} \mu_{M}(x)
$$

where $\mu_{M}$ is the Liouville measure $\omega^{n} / n$ !.

THEOREM 6.1. Assume the intersection of $\Gamma_{1}$ and $\Gamma_{2}$ is transversal and consists of a single point $y$, then

$$
\left(F_{1}^{k} a_{1}(\cdot, k), F_{2}^{k} a_{2}(\cdot, k)\right) \sim\left(\frac{2 \pi}{k}\right)^{n}\left(F_{1}(y), F_{2}(y)\right)_{L_{y}}^{k}\left(a_{1,0}(y), a_{2,0}(y)\right)_{T_{y} \Gamma_{1}, T_{y} \Gamma_{2}}
$$

where $(\cdot, \cdot)_{T_{y} \Gamma_{1}, T_{y} \Gamma_{2}}$ is the sesquilinear pairing $\delta_{y} \times \delta_{y} \rightarrow \mathbb{C}$ defined in section 6.1.

Proof. Let us write $\left(F_{1}(x), F_{2}(x)\right)_{L_{x}}=e^{i \varphi(x)}$. One has to estimate

$$
\int_{M} e^{i k \varphi(x)}\left(a_{1}(x, k), a_{2}(x, k)\right)_{\delta_{x}} \mu_{M}(x) .
$$

This will be an application of the stationnary phase lemma. First since $\left|F_{i}(x)\right|<1$ if $x \notin \Gamma_{i}$, the imaginary part of $\varphi(x)$ is positive if $x \notin \Gamma_{1} \cap \Gamma_{2}$. Next to compute the derivatives of the function $\varphi$, let us recall the content of lemma 4.2 of [5]. One has

$$
\nabla^{L} F_{i}=\frac{1}{i} \alpha_{i} \otimes F_{i}
$$

where $\alpha_{i}$ is a 1 -form vanishing along $\Gamma_{i}$. Furthermore for any vector fields $X$ and $Y$, one has at $x \in \Gamma_{i}$,

$$
\mathcal{L}_{X}\left\langle\alpha_{i}, Y\right\rangle(x)=\omega\left(q_{i} X(x), Y(x)\right)
$$


where $q_{i}$ is the projection of $T_{x} M \otimes \mathbb{C}$ onto $T_{x}^{0,1} M$ with kernel $T_{x} \Gamma_{i} \otimes \mathbb{C}$. One deduces from (27) that

$$
d \varphi=\bar{\alpha}_{2}-\alpha_{1} .
$$

So the point $y \in \Gamma_{1} \cap \Gamma_{2}$ is a critical point of $\varphi$ and one computes the Hessian at $y$ by using (28):

$$
\begin{aligned}
\operatorname{Hess} \varphi(X, Y) & =\mathcal{L}_{X}\left(\left(\bar{\alpha}_{2}-\alpha_{1}\right)(Y)\right) \\
& =\omega\left(\bar{q}_{2} X-q_{1} X, Y\right) \\
& =-i \omega\left(J\left(\bar{q}_{2}+q_{1}\right) X, Y\right) \\
& =i g\left(\left(\bar{q}_{2}+q_{1}\right) X, Y\right)
\end{aligned}
$$

where $g$ is the metric $g(X, Y)=\omega(X, J Y)$. At the third line, we used that $\bar{q}_{2} X \in$ $T_{y}^{1,0} M=\operatorname{ker}\left(J_{y}-i\right)$ whereas $q_{1} X \in T_{y}^{0,1} M=\operatorname{ker}\left(J_{y}+i\right)$. Since the intersection of $\Gamma_{1}$ and $\Gamma_{2}$ is transverse, the Hessian is non-degenerate. Then stationnary phase lemma (cf. [12] chapter 7.7) leads the following equivalent for the integral (26)

$$
\left(\frac{2 \pi}{k}\right)^{n} e^{i k \varphi(y)} \operatorname{det}^{-\frac{1}{2}}\left[-i \operatorname{Hess} \varphi\left(\partial_{x_{j}}, \partial_{x_{k}}\right)(y)\right]_{j, k=1, \ldots, 2 n}\left(a_{1,0}(y), a_{2,0}(y)\right)_{\delta_{y}} \rho(y)
$$

where $x_{1}, \ldots, x_{2 n}$ are local coordinates at $y$ and $\rho(y)$ is such that

$$
\mu_{M}(y)=\rho(y) d x_{1} \wedge \ldots \wedge d x_{2 n} .
$$

Here the square root of the determinant is determined on the space of symmetric complex matrices with a positive real part in such a way that it is positive on the subset of real matrices. The Liouville measure $\omega^{n} / n$ ! is also the Riemannian measure of $g$, so that

$$
\rho(y)=\operatorname{det}^{\frac{1}{2}}\left[g\left(\partial_{x_{j}}, \partial_{x_{k}}\right)(y)\right]_{j, k=1, \ldots, 2 n} .
$$

Then it follows from (29) that

$$
\operatorname{det}\left[-i \operatorname{Hess} \varphi\left(\partial_{x_{j}}, \partial_{x_{k}}\right)(y)\right]_{j, k=1, \ldots, 2 n} \rho^{-2}(y)=\operatorname{det}\left(\bar{q}_{2}+q_{1}\right) .
$$

Finally the determinant of $\bar{q}_{2}+q_{1}$ is easily computed in terms of the map $A: J T_{y} \Gamma_{1} \rightarrow$ $J T_{y} \Gamma_{1}$ such that $T_{y} \Gamma_{2}$ is the graph of $J A: J T_{y} \Gamma_{1} \rightarrow T_{y} \Gamma_{1}$. One has

$$
\operatorname{det}\left(\bar{q}_{2}+q_{1}\right)=\frac{2^{n}}{\operatorname{det}(1-i A)} .
$$

Comparing with lemma 6.1, we get the final result.

7. Asymptotics of $6 j$-symbols. Assume that $n=4$ and choose $\left(\ell_{i}\right)_{i=1, \ldots, 4}$ satisfying condition (10) and (11). The moduli space

$$
\mathcal{M}_{\ell}=\left(S_{\ell_{1}}^{2} \times S_{\ell_{2}}^{2} \times S_{\ell_{3}}^{2} \times S_{\ell_{4}}^{2}\right) / / S U(2)
$$

is a 2-dimensional sphere. Let $\Gamma$ be the left graph of figure 7 . Denote by $H_{k \ell-1}$ the operator associated to its internal edge and by $h \in \mathcal{C}^{\infty}\left(\mathcal{M}_{\ell}\right)$ the associated symbol

$$
h\left(\left[u_{1}, u_{2}, u_{3}, u_{4}\right]\right)=\left|u_{1}+u_{2}\right|^{2}, \quad\left(u_{1}, u_{2}, u_{3}, u_{4}\right) \in S_{\ell_{1}}^{2} \times \ldots \times S_{\ell_{4}}^{2} .
$$




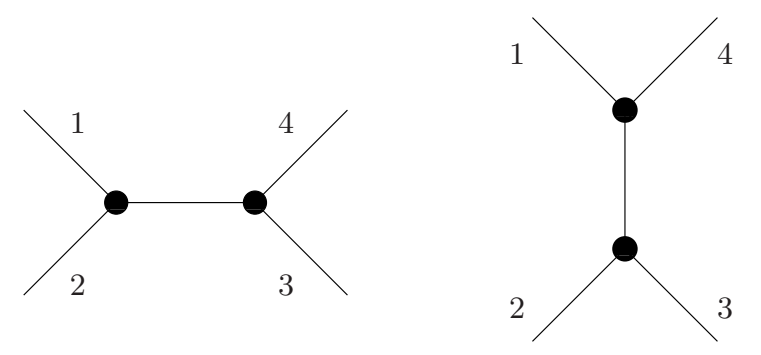

Fig. 7. The graphs $\Gamma$ and $\Gamma^{\prime}$

Then $h\left(\mathcal{M}_{\ell}\right)=[m, M]$ where

$$
m=\max \left(\left|\ell_{1}-\ell_{2}\right|,\left|\ell_{3}-\ell_{4}\right|\right), \quad M=\min \left(\ell_{1}+\ell_{2}, \ell_{3}+\ell_{4}\right) .
$$

The fibres of $h$ are circles except for the two singular ones $h^{-1}(m)$ and $h^{-1}(M)$ which consist of one point.

Consider now the right graph $\Gamma^{\prime}$ of figure 7 and denote by $H_{k \ell-1}^{\prime}$ and $h^{\prime}$ the associated operator and symbol. Introduce two orthonormal bases $\left(\Psi_{E, k}\right)_{E}$ and $\left(\Psi_{E, k}^{\prime}\right)_{E}$ of eigenvectors of $H_{k \ell-1}$ and $H_{k \ell-1}^{\prime}$ respectively.

$$
\frac{1}{k^{2}} H_{k \ell-1} \Psi_{E, k}=E \Psi_{E, k}, \quad \frac{1}{k^{2}} H_{k \ell-1}^{\prime} \Psi_{E, k}^{\prime}=E \Psi_{E, k}^{\prime} .
$$

By the results of part 5.1 these eigenvectors are Lagrangian sections associated to the level sets of $h$ and $h^{\prime}$. From this one can deduce the asymptotics of the scalar product

$$
\left(\Psi_{E, k}, \Psi_{E^{\prime}, k}^{\prime}\right)
$$

when $h^{-1}(E)$ and $h^{-1}\left(E^{\prime}\right)$ intersect transversally, by applying theorem 6.1.

7.1. The result. Assume first that $h^{-1}\left(E_{0}\right)$ and $h^{-1}\left(E_{0}^{\prime}\right)$ do not intersect. Then it follows from theorem 5.1 that there exist neighborhoods $V$ and $V^{\prime}$ of $E_{0}$ and $E_{0}^{\prime}$ respectively and a sequence $\left(C_{N}\right)$ such that

$$
\left|\left(\Psi_{E, k}, \Psi_{E^{\prime}, k}^{\prime}\right)\right| \leqslant C_{N} k^{-N}, \quad \forall N, \forall k
$$

and for any eigenvalues $E \in \operatorname{Sp}\left(k^{-2} H_{k \ell-1}\right) \cap V$ and $E^{\prime} \in \operatorname{Sp}\left(k^{-2} H_{k \ell-1}^{\prime}\right) \cap V^{\prime}$.

Next to understand better the possible configurations of the level sets of $h$ and $h^{\prime}$, it is useful to think of $\mathcal{M}_{\ell}$ as a space of tetrahedra, the point $\left[u_{1}, u_{2}, u_{3}, u_{4}\right]$ representing the tetrahedron $x$ of $\mathfrak{s u}(2)$ with vertices

$$
0, u_{1}, u_{1}+u_{2}, u_{1}+u_{2}+u_{3} .
$$

Furthermore two tetrahedra are identified if there are related by an orientationpreserving isometry. The lengths of the edges of the tetrahedron $x$ are

$$
\sqrt{h(x)}, \sqrt{h^{\prime}(x)}, \ell_{1}, \ell_{2}, \ell_{3} \text { and } \ell_{4} .
$$

The subset $C$ of coplanar tetrahedra is an embedded circle. It contains the four points where $h$ and $h^{\prime}$ attain their maximum and minimum. At the other points of $C$, the fibres of $h$ and $h^{\prime}$ are tangent. Outside of this circle, the fibres intersect transversally, cf. figure 8 . 


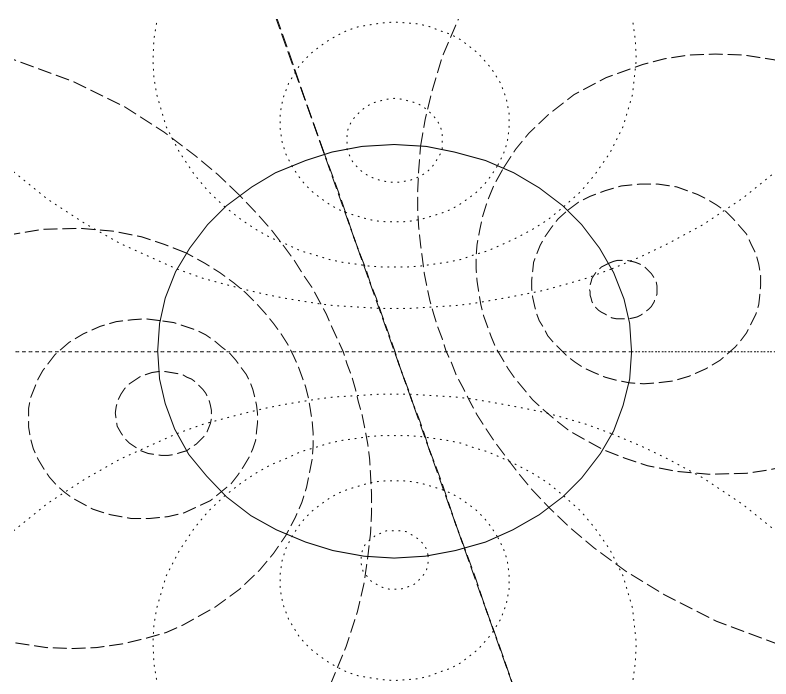

FIG. 8. The two fibrations and the circle $C$

Hence if $\sqrt{E}, \sqrt{E^{\prime}}, \ell_{1}, \ell_{2}, \ell_{3}$ and $\ell_{4}$ are the edge lengths of a non-degenerate tetrahedron $\tau$, then $h^{-1}(E)$ and $h^{\prime-1}\left(E^{\prime}\right)$ intersect transversally at two points represented by $\tau$ and its mirror image $\bar{\tau}$. Denote by $V\left(E, E^{\prime}\right)$ the volume of these tetrahedra and by $\theta\left(E, E^{\prime}\right)$ the sum

$$
\theta\left(E, E^{\prime}\right)=\alpha \sqrt{E}+\alpha^{\prime} \sqrt{E^{\prime}}+\sum_{i=1, \ldots, 4} \alpha_{i} \ell_{i}
$$

where $\alpha, \alpha^{\prime}, \alpha_{1}, \alpha_{2}, \alpha_{3}$ and $\alpha_{4}$ are the exterior dihedral angles (the exterior dihedral angle at an edge is the angle in $[0, \pi]$ between the outward normal vectors of the faces meeting at the edge).

Theorem 7.1. Assume that $\sqrt{E_{0}}, \sqrt{E_{0}^{\prime}}, \ell_{1}, \ell_{2}, \ell_{3}$ and $\ell_{4}$ are the edge lengths of a non-degenerate tetrahedron. Then there exist neighborhoods $V$ and $V^{\prime}$ of $E_{0}$ and $E_{0}^{\prime}$ respectively such that for every $k$ and for any eigenvalues $E \in \operatorname{Sp}\left(k^{-2} H_{k \ell-1}\right) \cap V$ and $E^{\prime} \in \operatorname{Sp}\left(k^{-2} H_{k \ell-1}^{\prime}\right) \cap V^{\prime}$, one has

$$
\left(\Psi_{E, k}, \Psi_{E^{\prime}, k}^{\prime}\right)=C_{k, E, E^{\prime}} \sqrt{\frac{2}{3 \pi}} k^{-\frac{1}{2}} \frac{\left(E E^{\prime}\right)^{\frac{1}{4}}}{V\left(E, E^{\prime}\right)^{\frac{1}{2}}} \cos \left(k \theta\left(E, E^{\prime}\right) / 2+\pi / 4\right)+O\left(k^{-\frac{3}{2}}\right)
$$

where the $O$ is uniform with respect to $E$ and $E^{\prime}$ and the $C_{k, E, E^{\prime}}$ are complex numbers of modulus 1.

This result is proved in part 7.2. The volume $V\left(E, E^{\prime}\right)$ and the function $\theta\left(E, E^{\prime}\right)$ appear in the computation of a holonomy and a symplectic product, as it was already understood in [19].

The $6 j$-symbols are defined in terms of this scalar product by a minor renormalisation

$$
\left\{\begin{array}{lll}
k \ell_{1}-1 & k \ell_{2}-1 & k \ell-1 \\
k \ell_{3}-1 & k \ell_{4}-1 & k \ell^{\prime}-1
\end{array}\right\}=(-1)^{k\left(\ell_{1}+\ell_{2}+\ell_{3}+\ell_{4}\right) / 2} \frac{\left(\Psi_{E, k}, \Psi_{E^{\prime}, k}^{\prime}\right)}{k \sqrt{\ell \ell^{\prime}}}
$$




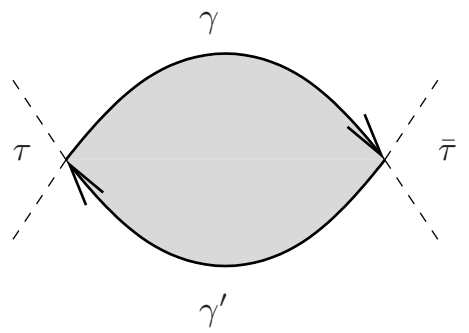

FIG. 9. The domain $D\left(E, E^{\prime}\right)$

with $E=\ell^{2}-k^{-2}$ and $E^{\prime}=\ell^{\prime 2}-k^{-2}$. Here we assume that the bases $\left(\Psi_{E, k}\right)$ and $\left(\Psi_{E^{\prime}, k}^{\prime}\right)$ are suitably defined and not only up to a phase factor. If $\ell, \ell^{\prime}, \ell_{1}, \ell_{2}, \ell_{3}$ and $\ell_{4}$ are the edge lengths of a non degenerate tetrahedron, we obtain

$$
\left\{\begin{array}{lll}
k \ell_{1}-1 & k \ell_{2}-1 & k \ell-1 \\
k \ell_{3}-1 & k \ell_{4}-1 & k \ell^{\prime}-1
\end{array}\right\} \sim \sqrt{\frac{2}{3 \pi}} k^{-\frac{3}{2}} V\left(\ell^{2}, \ell^{\prime 2}\right)^{-\frac{1}{2}} \cos \left(k \theta\left(\ell^{2}, \ell^{\prime 2}\right) / 2+\pi / 4\right)
$$

up to a phase factor, which is in agreement with the result of Roberts in [17].

7.2. Proof of theorem 7.1. Consider a non-degenerate tetrahedron $\tau$ with edge lengths $\sqrt{E}, \sqrt{E^{\prime}}, \ell_{1}, \ell_{2}, \ell_{3}$ and $\ell_{4}$. Denote by $\bar{\tau}$ its mirror image. Then the circle $h^{-1}(E)$ is the union of two segments delimited by $\tau$ and $\bar{\tau}$. On one of these segments, $h^{\prime}$ takes larger value than $E^{\prime}=h^{\prime}(\tau)=h^{\prime}(\bar{\tau})$. We shall denote it by $\gamma$ and orientate it according to the Hamiltonian flow of $h$. Consider in the same way the oriented segment $\gamma^{\prime} \subset h^{\prime-1}\left(E^{\prime}\right)$. Then interchanging $\tau$ and $\bar{\tau}$ if necessary, one has

$$
\partial \gamma=\bar{\tau}-\tau \quad \text { and } \quad \partial \gamma^{\prime}=\tau-\bar{\tau}
$$

Furthermore $\gamma \cup \gamma^{\prime}$ divides the sphere $\mathcal{M}_{\ell}$ in two domains. Let $D\left(E, E^{\prime}\right)$ be the one which does not contain $h^{-1}(E) \backslash \gamma$ and $h^{\prime-1}\left(E^{\prime}\right) \backslash \gamma^{\prime}$, cf. figure 9 . We oriente it in such a way that the the symplectic area $\int_{D\left(E, E^{\prime}\right)} \omega$ is positive. The oriented boundary of $D\left(E, E^{\prime}\right)$ is then $-\gamma \cup-\gamma^{\prime}$.

Then adapting the results of [19] on spherical tetrahedra to the simpler Euclidean case, we prove the following

Proposition 7.1. The symplectic area of $D\left(E, E^{\prime}\right)$ is

$$
A\left(E, E^{\prime}\right)=-\theta\left(E, E^{\prime}\right)+\pi\left(\ell_{1}+\ell_{2}+\ell_{3}+\ell_{4}\right)
$$

and

$$
\left.\omega\left(X, X^{\prime}\right)\right|_{\tau}=-\left.\omega\left(X, X^{\prime}\right)\right|_{\bar{\tau}}=3 \frac{V\left(E, E^{\prime}\right)}{\sqrt{E E^{\prime}}}
$$

where $X$ and $X^{\prime}$ are the Hamiltonian vector fields of $\frac{1}{2} \sqrt{h}$ and $\frac{1}{2} \sqrt{h^{\prime}}$ respectively and $\theta\left(E, E^{\prime}\right)$ has been defined in (30).

By theorem 5.2, modifying the Lagrangian sections $\Psi_{E, k}$ and $\Psi_{E^{\prime}, k^{\prime}}^{\prime}$ by phase factors if necessary, one has on a neighborhood of $D\left(E, E^{\prime}\right)$,

$$
\begin{aligned}
\Psi_{E, k} & =\left(\frac{k}{2 \pi}\right)^{\frac{1}{4}} F^{k}(E, \cdot) g(E, \cdot k)+O\left(k^{-\infty}\right), \\
\Psi_{E, k}^{\prime} & =\left(\frac{k}{2 \pi}\right)^{\frac{1}{4}} F^{\prime k}(E, \cdot) g^{\prime}(E, \cdot, k)+O\left(k^{-\infty}\right) .
\end{aligned}
$$


Then by theorem 6.1 , one has

$$
\left(\Psi_{E, k}, \Psi_{E^{\prime}, k}\right) \sim\left(\frac{2 \pi}{k}\right)^{\frac{1}{2}} \sum_{x=\tau, \bar{\tau}}\left(F(E, x), F^{\prime}\left(E^{\prime}, x\right)\right)_{L_{x}}^{k}\left(g_{0}(E, x), g_{0}^{\prime}\left(E^{\prime}, x\right)\right)_{E, E^{\prime}, x}
$$

Here $(\cdot, \cdot)_{E, E^{\prime}, x}$ is the sesquilinear pairing of $\delta_{x}$ defined on part 6.1 with the Lagrangian subspaces $T_{x} h^{-1}(E)$ and $T_{x} h^{\prime-1}\left(E^{\prime}\right)$ of $T_{x} \mathcal{M}_{\ell}$. Denote by $\varphi$ the isomorphism between $\delta_{x}^{2}$ and $\wedge^{1,0} T_{x}^{*} \mathcal{M}_{\ell}$. By definition of the pairing one has

$$
\left(s, s^{\prime}\right)_{E, E^{\prime}, x}^{2}=i \frac{\varphi\left(s^{2}\right)(e) \overline{\varphi\left(s^{\prime 2}\right)\left(e^{\prime}\right)}}{\omega\left(e, e^{\prime}\right)}, \quad \forall s, s^{\prime} \in \delta_{x}
$$

for any nonvanishing vectors $e \in T_{x} h^{-1}(E)$ and $e^{\prime} \in T_{x} h^{-1}\left(E^{\prime}\right)$. It follows from condition 4. of theorem 5.2 that for $x=\tau$ or $\bar{\tau}$, one has

$$
\varphi\left(g_{0}^{2}(E, x)\right)(X(x))=\varphi\left(g_{0}^{\prime 2}\left(E^{\prime}, x\right)\right)\left(X^{\prime}(x)\right)=\frac{1}{2 \pi}
$$

where $X$ and $X^{\prime}$ are the Hamiltonian vector fields of $\frac{1}{2} \sqrt{h}$ and $\frac{1}{2} \sqrt{h^{\prime}}$. Then we deduce from (32) that

$$
\left(g_{0}(E, x), g_{0}^{\prime}\left(E^{\prime}, x\right)\right)_{E, E^{\prime}, x}=\frac{1}{2 \pi} \frac{e^{i \pi / 4}}{\sqrt{\left.\omega\left(X, X^{\prime}\right)\right|_{x}}}
$$

with the suitable determination of the square root. So we deduce from the second part of proposition 7.1 that both terms of the sum in equation (31) has the same modulus. We will prove that their phase difference is

$$
k\left(\theta\left(E, E^{\prime}\right)-\pi\left(\ell_{1}+\ell_{2}+\ell_{3}+\ell_{4}\right)\right)+\pi / 2 .
$$

Taking into account that $\ell_{1}+\ell_{2}+\ell_{3}+\ell_{4}$ is an even integer, this will end the proof of theorem 7.1.

Since $F(E, \cdot)$ and $F^{\prime}\left(E^{\prime}, \cdot\right)$ are flat along $\gamma$ and $\gamma^{\prime}$ respectively, one has

$$
\left(F(E, \bar{\tau}), F^{\prime}\left(E^{\prime}, \bar{\tau}\right)\right)_{L_{\tau}}=H\left(E, E^{\prime}\right)\left(F(E, \tau), F^{\prime}\left(E^{\prime}, \tau\right)\right)_{L_{\bar{\tau}}}
$$

where $H\left(E, E^{\prime}\right) \in \mathbb{C}$ is the holonomy of $L$ along $\gamma \cup \gamma^{\prime}$. Since $L$ has curvature $\frac{1}{i} \omega$ and $-\left(\gamma \cup \gamma^{\prime}\right)$ is the boundary of $D\left(E, E^{\prime}\right)$, one has

$$
H\left(E, E^{\prime}\right)=\exp \left(-i A\left(E, E^{\prime}\right)\right)
$$

where $A\left(E, E^{\prime}\right)$ is the symplectic area computed in proposition 7.1. Furthermore it follows from equation (34) that

$$
\left(g_{0}(E, \bar{\tau}), g_{0}^{\prime}\left(E^{\prime}, \bar{\tau}\right)\right)_{E, E^{\prime}, \bar{\tau}}=e^{ \pm i \pi / 2}\left(g_{0}(E, \tau), g_{0}^{\prime}\left(E^{\prime}, \tau\right)\right)_{E, E^{\prime}, \tau} .
$$

It happens that the undetermined sign is positive and the proof relies uniquely on the configuration of the level sets, as they appear in figure 9.

To see this, trivialise the tangent bundle of $\mathcal{M}_{\ell}$ on a neighborhood of $D\left(E, E^{\prime}\right)$ in such a way that the vector fields $X / \sqrt{\omega(X, J X)}$ and $J X / \sqrt{\omega(X, J X)}$ are send to constant vectors that we denote by $e$ and $f$. The symplectic and complex structures are constant in this trivialisation

$$
\omega(e, f)=1, \quad J e=f .
$$




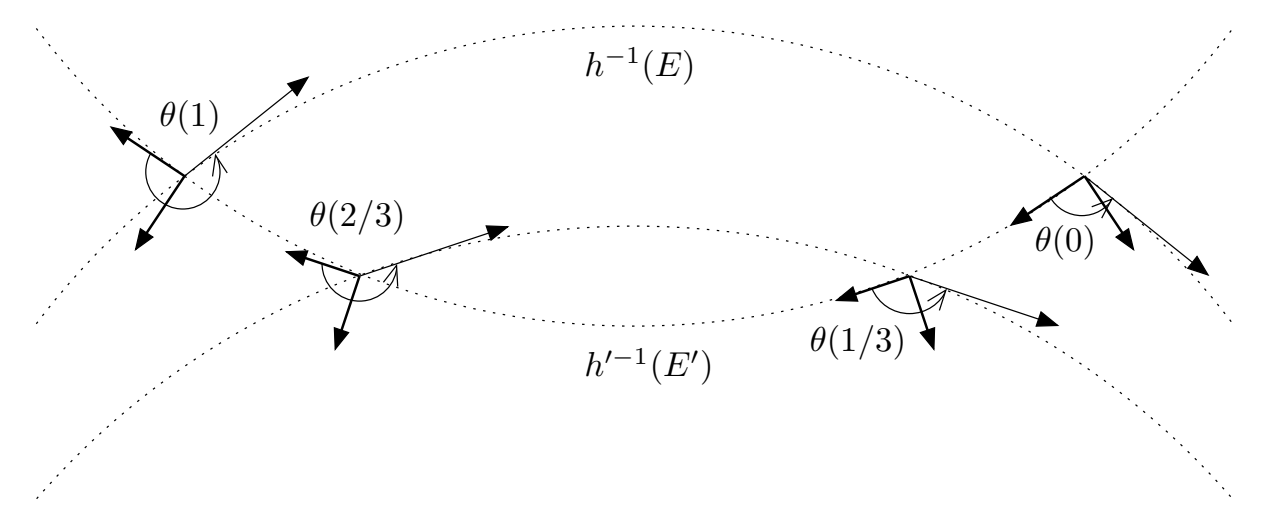

FIG. 10. the angle $\theta$

Trivialise also the half-form bundle, in such a way that the constant half-form $s$ squares to $e^{*}+i f^{*}$, where $\left(e^{*}, f^{*}\right)$ is the dual base of $(e, f)$. Then one may explicitely compute the sesquilinear pairing associated to the Lagrangian lines generated by $e$ and $f_{\theta}=(\cos \theta) f-(\sin \theta) e$. By formula (32) we have

$$
(s, s)_{\mathbb{R} e, \mathbb{R} f_{\theta}}^{2}=\frac{e^{-i \theta}}{\cos \theta} .
$$

Choosing the determination of the square root as in part 6.1 , we obtain for $\theta \in$ $(-\pi / 2, \pi / 2)$,

$$
(s, s)_{\mathbb{R} e, \mathbb{R} f_{\theta}}=\frac{e^{-i \theta / 2}}{\sqrt{\cos \theta}} .
$$

Next introduce a parametrisation $x(t)$ of $\gamma$ with $x(0)=\tau$ and $x(1)=\bar{\tau}$. Then

$$
X(x(t))=|X(x(t))| e
$$

where $|X|=\sqrt{\omega(X, J X)}$. And modifying the sign of $g_{0}$ if necessary, one deduce from equation (33) that

$$
g_{0}(E, x(t))=(2 \pi|X(x(t))|)^{-\frac{1}{2}} s .
$$

Parametrize $-\gamma^{\prime}$ by $y(t)$ with $y(0)=\tau$ and $y(1)=\bar{\tau}$. Then the configuration (cf. figure 10) of the level sets of $h$ and $h^{\prime}$ implies that

$$
X^{\prime}(y(t))=r(t)((\cos \theta(t)) e+(\sin \theta(t)) f)
$$

where $r$ is a positive function and $\theta$ takes its values in $(0,2 \pi)$ with $\theta(0) \in(0, \pi)$ and $\theta(1) \in(\pi, 2 \pi)$. By equation (33)

$$
g_{0}^{\prime}\left(E^{\prime}, y(t)\right)=(2 \pi r(t))^{-\frac{1}{2}} e^{-i \theta(t) / 2} s .
$$

Finally the angle between the lines generated by $X(\tau)$ and $X^{\prime}(\tau)$ being $\theta(0)-\pi / 2$, we deduce from equations (35), (36) and (37) that

$$
\begin{aligned}
\left(g_{0}(E, \tau), g_{0}^{\prime}\left(E^{\prime}, \tau\right)\right)_{E, E^{\prime}, \tau} & =\frac{e^{-i\left(\frac{\theta(0)}{2}-\frac{\pi}{4}\right)}}{\sqrt{\cos (\theta(0)-\pi / 2)}} \cdot \frac{e^{i \frac{\theta(0)}{2}}}{2 \pi \sqrt{|X(\tau)| r(0)}} \\
& =\frac{e^{i \pi / 4}}{2 \pi}\left(\cos (\theta(0)-\pi / 2)|X(\tau)|\left|X^{\prime}(\tau)\right|\right)^{-\frac{1}{2}} .
\end{aligned}
$$


In the same way we obtain that

$$
\begin{aligned}
\left(g_{0}(E, \bar{\tau}), g_{0}^{\prime}\left(E^{\prime}, \bar{\tau}\right)\right)_{E, E^{\prime}, \bar{\tau}} & =\frac{e^{-i\left(\frac{\theta(1)}{2}-\frac{3 \pi}{4}\right)}}{\sqrt{\cos (\theta(1)-3 \pi / 2)}} \cdot \frac{e^{i \frac{\theta(1)}{2}}}{2 \pi \sqrt{|X(\tau)| r(1)}} \\
& =\frac{e^{i 3 \pi / 4}}{2 \pi}\left(\cos (\theta(1)-3 \pi / 2)|X(\bar{\tau})|\left|X^{\prime}(\bar{\tau})\right|\right)^{-\frac{1}{2}} .
\end{aligned}
$$

So the phase difference between the two pairings is $\pi / 2$.

\section{Semi-classical reduction with subprincipal estimates.}

8.1. Quantum reduction. Let $(M, \omega)$ be a connected compact Kähler manifold $(M, \omega)$ with a prequantization bundle $L \rightarrow M$ with curvature $\frac{1}{i} \omega$ and a half-form bundle $(\delta, \varphi)$. Here we denote by $\varphi$ the line bundle isomorphism $\delta^{2} \rightarrow \wedge^{\text {top }, 0} T^{*} M$. By assumption it preserves both the Hermitian and holomorphic structures. Let $G$ be a compact connected Lie group acting on $M$ in a Hamiltonian way. Denote by $\mu: M \rightarrow \mathfrak{g}^{*}$ the moment map. We assume that the action lifts to the prequantization bundle in such a way that the infinitesimal action on sections of $L$ is given by

$$
\nabla_{\xi^{\#}}+i \mu^{\xi}, \quad \forall \xi \in \mathfrak{g} .
$$

We assume furthermore that the action preserves the complex structure and lifts to the half-form bundle in such a way that $\varphi$ is equivariant. Under these assumptions, the group $G$ acts naturally on the space $H^{0}\left(M, L^{k} \otimes \delta\right)$ for any positive integer $k$, the infinitesimal action being given by the Kostant-Souriau operators (7). We denote by

$$
H_{G}^{0}\left(M, L^{k} \otimes \delta\right)
$$

the $G$-invariant subspace.

Suppose that $G$ acts freely on the zero-set $P:=\mu^{-1}(0)$. Then 0 is a regular value of the moment, $P$ is a coisotropic submanifold of $M$ and its characteristic distribution is the tangent space to the orbits. So the quotient

$$
M_{r}:=P / G
$$

is a symplectic manifold. Consider the quotient $L_{r}$ of the restriction of $L$ to $P$ by the $G$-action. Since the action preserves the connection of $L$ and is by parallel transport over $P, L_{r}$ inherits a connection. Its curvature is $\frac{1}{i} \omega_{r}$ where $\omega_{r}$ is the reduced symplectic form.

To define the complex structure on the symplectic quotient, introduce the complexification $G^{\mathbb{C}}$ of $G$. It is a complex connected Lie group containing $G$ as a maximal compact subgroup. The Lie algebra of $G^{\mathbb{C}}$ is the complexification of $\mathfrak{g}$ and the Cartan decomposition is the diffeomorphism

$$
G^{\mathbb{C}} \simeq \exp (i \mathfrak{g}) G
$$

Furthermore the set $\exp (i \mathfrak{g})$ is diffeomorphic to the vector space $\mathfrak{g}$, the diffeomorphism being the exponential map.

The $G$-action extends to a holomorphic action of $G^{\mathbb{C}}$ whose infinitesimal action is given by

$$
\xi^{\#}+J \eta^{\#}, \quad \forall \xi+i \eta \in \mathfrak{g} \oplus i \mathfrak{g}=\mathfrak{g} \otimes \mathbb{C} .
$$


The saturated set $M_{s}:=G^{\mathbb{C}}$.P of the zero set of $\mu$ is called the stable set. Since the vector field $J \xi^{\#}$ is the Riemannian gradient of $\mu^{\xi}$ for any vector $\xi, M_{s}$ is an open set diffeomorphic to $\mathfrak{g} \times P$, the diffeomorphism being

$$
\mathfrak{g} \times P \rightarrow M_{s}, \quad(\xi, x) \rightarrow \exp (i \xi) \cdot x .
$$

Furthermore the action of $G$ on $P$ being free, the action of $G^{\mathbb{C}}$ on $M_{s}$ is also free. Finally the injection of $P$ into $M$ induces a diffeomorphism

$$
P / G \simeq M_{s} / G^{\mathbb{C}}
$$

In this way the symplectic quotient inherits a complex structure. It is compatible with the symplectic form and $M_{r}$ becomes a Kähler manifold.

Similarly the $G$-action on the prequantization bundle and the half-form bundle can be analytically continued to holomorphic actions of the complexified group $G^{\mathbb{C}}$. We have a natural identification between $L_{r}$ and the quotient by $G^{\mathbb{C}}$ of the restriction of $L$ to the stable set. Hence $L_{r}$ inherits a holomorphic structure, it is compatible with the connection. We define $\delta_{r}$ as the quotient by $G^{\mathbb{C}}$ of the restriction of $\delta$ to $M_{s}$. This is a holomorphic line bundle on $M_{r}$. The holomorphic $G$-invariant sections of $L^{k} \otimes \delta$ are also invariant under the complexified action. This defines a natural map

$$
V_{k}: H_{G}^{0}\left(M, L^{k} \otimes \delta\right) \rightarrow H^{0}\left(M_{r}, L_{r}^{k} \otimes \delta_{r}\right) .
$$

Theorem 8.1 (Guillemin-Sternberg). When $k$ is sufficiently large, $V_{k}$ is an isomorphism.

The condition on $k$ is due to the presence of the half-form bundle. It does not appear in theorem 3.1 because in this case the half-form bundle is a power of the prequantum bundle.

The theorem says that the $G^{\mathbb{C}}$-equivariant holomorphic sections of $L^{k} \otimes \delta$ over the stable set extend uniquely into $G^{\mathbb{C}}$-equivariant holomorphic sections over $M$. This follows from the non-trivial fact that the complementary of the stable set is contained in a complex submanifold of codimension $\geqslant 1$. Furthermore the $G^{\mathbb{C}}$-equivariant sections of $L^{k} \otimes \delta \rightarrow M_{s}$ are bounded when $k$ is sufficiently large. In the next section we will prove an explicit estimate that we will use in the sequel.

8.2. Estimates of the equivariant sections. Introduce a norm $\|\cdot\|$ on the Lie algebra $\mathfrak{g}$.

Proposition 8.1. There exists $C_{1}, C_{2}>0$ such that for any integer $k$ and any $G^{\mathbb{C}}$-equivariant section $\Psi$ of $L^{k} \otimes \delta \rightarrow M_{s}$, one has

$$
\|\Psi(\exp (i \xi) \cdot x)\|^{2} \leqslant e^{C_{1}\|\xi\|-k C_{2}\|\xi\|^{2}}\|\Psi(x)\|^{2}, \quad \forall x \in P, \forall \xi \in \mathfrak{g}
$$

where we denote by $\|\cdot\|$ the pointwise norm of $L^{k} \otimes \delta$.

Proof. We start by estimating $\|\exp (i \xi) \cdot u\|$ in terms of $\|u\|$ for any $u \in \delta$. Introduce the smooth function $r$ on $\mathfrak{g} \times M$

$$
r(\xi, x):=\left.\frac{d}{d t}\right|_{t=0} \frac{\|\exp (i t \xi) \cdot u\|^{2}}{\|u\|^{2}}, \quad \text { where } u \in \delta_{x} .
$$

We have

$$
\frac{d}{d t}\|\exp (i t \xi) \cdot u\|^{2}=r(\xi, \exp (i t \xi) \cdot x)\|\exp (i t \xi) \cdot u\|^{2}
$$


Let $C_{1}$ be the supremum of $|r|$ on the compact set $\{\|\xi\|=1\} \times M$. Then by integrating the previous equality we get for $\|\xi\|=1$ that

$$
\|\exp (i t \xi) \cdot u\|^{2} \leqslant e^{C_{1}|t|}\|u\|^{2} .
$$

Let us now estimate $\|\exp (i \xi) . u\|$ in terms of $\|u\|$ for any $u \in L$. First we have

$$
\left.\frac{d}{d t}\right|_{t=0} \frac{\|\exp (i t \xi) \cdot u\|^{2}}{\|u\|^{2}}=2 \mu^{\xi}(x), \quad \text { if } u \in L_{x} .
$$

This follows from the fact that the infinitesimal action of $i \xi \in \mathfrak{g} \otimes \mathbb{C}$ on the sections of $L$ is $\nabla_{J \xi^{\#}}-\mu^{\xi}$. Furthermore since $J \xi^{\#}$ is the gradient of $\mu^{\xi}$, we have

$$
\frac{d}{d t} \mu^{\xi}(\exp (i t \xi) \cdot x)=-g\left(\xi^{\#}, \xi^{\#}\right)(\exp (i t \xi) \cdot x)
$$

where $g$ is the Riemannian metric $g(X, Y)=\omega(X, J Y)$ of $M$. Let $C_{2}$ be the infimum of $g\left(\xi^{\#}, \xi^{\#}\right)(x)$ on the compact $\{\|\xi\|=1\} \times M$. By integrating, we obtain for $\|\xi\|=1$

$$
\mu^{\xi}(\exp (i t \xi) \cdot x) \leqslant \mu^{\xi}(x)-C_{2} t
$$

Integrating again we obtain for $x \in P$ (and consequently $\mu^{\xi}(x)=0$ ) that

$$
\|\exp (i t \xi) \cdot u\|^{2} \leqslant e^{-C_{2} t^{2}}\|u\|^{2}, \quad \forall u \in L_{x} .
$$

This ends the proof.

8.3. The reduced half-form bundle. In this part we define the isomorphism $\varphi_{r}: \delta_{r}^{2} \rightarrow \wedge^{\mathrm{top}, 0} T^{*} M_{r}$ which makes $\delta_{r}$ a half-form bundle. Recall that the isomorphism $\delta \rightarrow \wedge^{\text {top }, 0} T^{*} M$ was denoted by $\varphi$. Let $\pi_{s}$ be the projection from the stable set $M_{s}$ onto the quotient $M_{r}=M_{s} / G^{\mathbb{C}}$. Introduce the bundle over $M_{s}$

$$
E=\left(\operatorname{ker}\left(\pi_{s}\right)_{\star} \otimes \mathbb{C}\right) \cap T^{1,0} M_{s}
$$

One has an exact sequence

$$
0 \rightarrow E \rightarrow T^{1,0} M_{s} \rightarrow \pi_{s}^{*} T^{1,0} M_{r} \rightarrow 0,
$$

Consider an invariant metric (., .) on the Lie algebra $\mathfrak{g}$ and an orthonormal base $\left(\xi_{i}\right)$ of $\mathfrak{g}$. Define the section of $\wedge^{\text {top }} E$

$$
\gamma=\frac{1}{2^{\ell}}\left(\xi_{1}^{\#}-i J \xi_{1}^{\#}\right) \wedge \ldots \wedge\left(\xi_{\ell}^{\#}-i J \xi_{\ell}^{\#}\right) .
$$

This section $\gamma$ does not depend on the choice of the base $\left(\xi_{i}\right)$. It does not vanish, is holomorphic and $G^{\mathbb{C}}$-equivariant. The contraction by $\gamma$ defines an $G^{\mathbb{C}}$-equivariant isomorphism

$$
\wedge^{\mathrm{top}, 0} T^{*} M_{s} \rightarrow \pi_{s}^{*} \wedge^{\mathrm{top}, 0} T^{*} M_{r}
$$

By composing with $\varphi$, we obtain an isomorphism from $\delta^{\otimes 2}$ to $\pi_{s}^{*} \wedge^{\text {top }, 0} T^{*} M_{r}$ which descends into

$$
\varphi_{r}: \delta_{r}^{\otimes 2} \rightarrow \wedge^{\mathrm{top}, 0} T^{*} M_{r}
$$


In other words, for any $u \in \delta$, one has

$$
\iota(\gamma) \varphi\left(u^{2}\right)=\pi_{s}^{*} \varphi_{r}\left([u]^{2}\right) .
$$

Observe that $\varphi_{r}$ is a holomorphic map.

We endow $\delta_{r}$ with the metric such that $\varphi_{r}$ becomes an isomorphism of Hermitian bundle. We have to be careful that the projection from $\left.\delta\right|_{M_{s}}$ onto $\delta_{r}$ does not preserve the metrics, even when it is restricted to the zero set of the moment map. For any $u, v \in \delta_{x}$ with $x$ in the zero level set,

$$
(u, v)_{\delta_{x}}=\|\gamma(x)\|^{-1}([u],[v])_{\delta_{r, x}} .
$$

And a straightforward computation gives the pointwise norm of $\gamma$

$$
\|\gamma(x)\|=2^{-\ell / 2} \operatorname{det}^{\frac{1}{2}}\left[g\left(\xi_{i}^{\#}, \xi_{j}^{\#}\right)\right]_{i, j=1, \ldots, \ell}(x)
$$

where $g$ is the metric $\omega(X, J Y)$. From now on, we assume that the invariant metric of $\mathfrak{g}$ is chosen so that the Riemannian volume of $G$ is 1 . This implies that the GuilleminSternberg isomorphism rescaled by a factor $(2 \pi / k)^{\ell / 4}$ is asymptotically unitary as was proved in [10]. This follows also from the next results, cf. the remark after theorem 8.4 .

8.4. A class of Fourier integral operators. We denote by $\pi$ the projection from the zero set $P$ of the moment map onto the quotient $M_{r}=P / G$. Let us introduce some datas associated to the symplectic reduction $M / / G$. First consider the submanifold

$$
\Lambda:=\{(x, \pi(x)), x \in P\} \subset M \times M_{r} .
$$

Denote by $M_{r}^{-}$the manifold $M_{r}$ endowed with the symplectic form $-\omega_{r}$. Then $\Lambda$ is a Lagrangian submanifold of $M \times M_{r}^{-}$called the moment Lagrangian. Next one defines a section $t$ of $L \otimes \bar{L}_{r}$ over $\Lambda$ by

$$
t(x, \pi(x))=u \otimes[\bar{u}]
$$

if $x \in P$ and $u \in L_{x}$ is a unitary vector. This section is flat and unitary. In a similar way consider the section $t_{\delta}$ of $\delta \otimes \bar{\delta}_{r}$ defined on $\Lambda$ by

$$
t_{\delta}(x, \pi(x))=u \otimes[\bar{u}]
$$

if $x \in P, u \in \delta_{x}$ and $[u] \in \delta_{r, x}$ is a unitary vector.

Next we define a space $\mathcal{F}$ of Fourier integral operators associated to $\left(\Lambda, t, t_{\delta}\right)$. First let us introduce the Schwartz kernel of an operator $\mathcal{H}_{r, k} \rightarrow \mathcal{H}_{k}$ where

$$
\mathcal{H}_{k}:=H^{0}\left(M, L^{k} \otimes \delta\right), \quad \mathcal{H}_{r, k}:=H^{0}\left(M_{r}, L_{r}^{k} \otimes \delta_{r}\right)
$$

The scalar product of $\mathcal{H}_{r, k}$ gives us an isomorphism

$$
\operatorname{End}\left(\mathcal{H}_{r, k}, \mathcal{H}_{k}\right) \simeq \mathcal{H}_{k} \otimes \overline{\mathcal{H}}_{r, k}
$$

The latter space can be regarded as the space of holomorphic sections of

$$
\left(L^{k} \otimes \delta\right) \otimes\left(\bar{L}_{r}^{k} \otimes \bar{\delta}_{r}\right) \rightarrow M \times \bar{M}_{r},
$$


where $\bar{M}_{r}$ is the manifold $M_{r}$ endowed with the conjugate complex structure. The section associated in this way to an operator is its Schwartz kernel.

Consider a sequence $\left(Q_{k}\right)$ such that for every $k, Q_{k}$ is an operator $\mathcal{H}_{r, k} \rightarrow \mathcal{H}_{k}$. We say that $\left(Q_{k}\right)$ is a Fourier integral operator of $\mathcal{F}$ if the sequence of Schwartz kernel satisfies

$$
Q_{k}(x, y)=\left(\frac{k}{2 \pi}\right)^{n_{r}+\frac{\ell}{4}} F^{k}(x, y) f(x, y, k)+O\left(k^{-\infty}\right)
$$

where

- $F$ is a section of $L \otimes \bar{L}_{r} \rightarrow M \times \bar{M}_{r}$ such that $\|F(x, z)\|<1$ if $(x, z) \notin \Lambda$,

$$
F(x, z)=t(x, z), \quad \forall(x, z) \in \Lambda
$$

and $\bar{\partial} F \equiv 0$ modulo a section vanishing to any order along $\Lambda$.

- $f(., k)$ is a sequence of sections of $\delta \otimes \bar{\delta}_{r} \rightarrow M \times \bar{M}_{r}$ which admits an asymptotic expansion in the $\mathcal{C}^{\infty}$ topology of the form

$$
f(., k)=f_{0}+k^{-1} f_{1}+k^{-2} f_{2}+\ldots
$$

whose coefficients satisfy $\bar{\partial} f_{i} \equiv 0$ modulo a section vanishing to any order along $\Lambda$.

Furthermore $n_{r}$ is the complex dimension of $M_{r}$ and $\ell$ is the dimension of $G$.

Let us define the principal symbol of $\left(Q_{k}\right)$ to be the function $g \in \mathcal{C}^{\infty}(P)$ such that the restriction to $\Lambda$ of the leading term $f_{0}$ of the previous asymptotic expansion is

$$
f_{0}(x, z)=g(x) t_{\delta}(x, z), \quad \forall(x, z) \in \Lambda .
$$

Denote by $\sigma: \mathcal{F} \rightarrow \mathcal{C}^{\infty}(P)$ the principal symbol map. Let $\mathcal{F}^{1}$ be the space of Fourier integral operator of order 1 , that is $\left(T_{k}\right) \in \mathcal{F}^{1}$ if and only if $\left(k T_{k}\right) \in \mathcal{F}$. The next result was proved in [4].

Theorem 8.2. The sequence $0 \rightarrow \mathcal{F}^{1} \rightarrow \mathcal{F} \stackrel{\sigma}{\rightarrow} \mathcal{C}^{\infty}(P) \rightarrow 0$ is exact.

8.5. Semiclassical properties of the reduction. Introduce the inverse of the Guillemin-Sternberg isomorphism (39)

$$
W_{k}: H^{0}\left(M_{r}, L_{r}^{k} \otimes \delta_{r}\right) \rightarrow H_{G}^{0}\left(M, L^{k} \otimes \delta\right) .
$$

Recall that we denote by $\ell$ the dimension of $G$.

THEOREM 8.3. The sequence $\left(\left(\frac{k}{2 \pi}\right)^{\frac{\ell}{4}} W_{k}\right)$ is an Fourier integral operator of $\mathcal{F}$ whose principal symbol is the constant function equal to 1.

We defined Toeplitz operator in section 3.3 with their principal and subprincipal symbols. In the following theorems we consider Toeplitz operators on $M$ and the reduced space $M_{r}$.

THEOREM 8.4. Let $\left(Q_{k}\right)$ and $\left(R_{k}\right)$ be Fourier integral of $\mathcal{F}$ with symbol $f_{Q}$ and $f_{R}$ respectively. Then $\left(Q_{k}^{*} R_{k}\right)$ is a Toeplitz operator of $M_{r}$. Its principal symbol is the function $g \in \mathcal{C}^{\infty}\left(M_{r}\right)$ given by

$$
g(z)=\int_{P_{z}} \overline{f_{Q}(x)} f_{R}(x) \mu_{P_{z}}(x), \quad \forall z \in M_{r}
$$


where $\mu_{P_{z}}$ is the $G$-invariant measure of $P_{z}=\pi^{-1}(z)$ normalized by $\int_{P_{z}} \mu_{P_{z}}=1$.

As a consequence of both theorems, $\left(\left(\frac{k}{2 \pi}\right)^{\frac{\ell}{2}} W_{k}^{*} W_{k}\right)$ is a Toeplitz operator with principal symbol 1. Hence its uniform norm is equivalent to 1 as $k$ tends to $\infty$. In other words, the Guillemin-Sternberg isomorphism rescaled by a factor $(2 \pi / k)^{\ell / 4}$ is asymptotically unitary, as it was already proved in [10]. Our final result is about the composition of Toeplitz operators with Fourier integral operators.

THEOREM 8.5. Let $\left(Q_{k}\right)$ be a Fourier integral operator of $\mathcal{F}$. Let $\left(S_{k}\right)$ and $\left(T_{k}\right)$ be Toeplitz operators of $M$ and $M_{r}$ respectively with principal symbols $f_{0}$ and $g_{0}$. Then the sequence $\left(S_{k} Q_{k} T_{k}\right)$ is a Fourier integral operator of $\mathcal{F}$ with symbol

$$
\left(j^{*} f_{0}\right) \sigma(Q)\left(\pi^{*} g_{0}\right)
$$

where $j$ is the injection $P \rightarrow M$ and $\pi$ the projection $P \rightarrow M_{r}$. Assume furthermore that $f_{0}$ is $G$-invariant and $j^{*} f_{0}=\pi^{*} g_{0}$, then

$$
S_{k} Q_{k}-Q_{k} T_{k}=k^{-1} R_{k}
$$

where $\left(R_{k}\right)$ is a Fourier integral operator of $\mathcal{F}$ with symbol

$$
\sigma\left(R_{k}\right)=\left(j^{*} f_{1}-\pi^{*} g_{1}+\frac{1}{i} \mathcal{L}_{X}\right) \sigma(Q)
$$

where $f_{1}$ and $g_{1}$ are the subprincipal symbols of $\left(S_{k}\right)$ and $\left(T_{k}\right)$ respectively and $X$ is the restriction to $P$ of the Hamiltonian vector field of $f_{0}$.

The previous theorem are proved in the following sections. Before let us deduce the result about the reduction of Toeplitz operators. Introduce the isomorphism of Hilbert spaces:

$$
U_{k}=W_{k}\left(W_{k}^{*} W_{k}\right)^{-\frac{1}{2}}: H^{0}\left(M_{r}, L_{r}^{k} \otimes \delta_{r}\right) \rightarrow H_{G}^{0}\left(M, L^{k} \otimes \delta\right) .
$$

As already noted, $\left(\left(\frac{k}{2 \pi}\right)^{\ell / 2} W_{k}^{*} W_{k}\right)$ is a Toeplitz operator with principal symbol the constant function equal to 1 . So the same holds for $\left(\left(\frac{k}{2 \pi}\right)^{-\ell / 4}\left(W_{k}^{*} W_{k}\right)^{-1 / 2}\right)$. Then theorem 8.5 implies that $\left(U_{k}\right)$ is a Fourier integral operator of $\mathcal{F}$ with symbol the constant function equal to 1 .

COROllary 8.1. Let $\left(S_{k}\right)$ be a Toeplitz operator of $M$ with principal symbol $f_{0}$. Then $\left(U_{k}^{*} S_{k} U_{k}\right)$ is a Toeplitz operator of $M_{r}$ whose principal symbol $g_{0}$ is given by

$$
g_{0}(z)=\int_{P_{z}} f_{0}(x) \mu_{P_{z}}(x)
$$

If furthermore $f_{0}$ is G-invariant, then the subprincipal symbol is

$$
g_{1}(z)=\int_{P_{z}} f_{1}(x) \mu_{P_{z}}(x)
$$

where $f_{1}$ is the subprincipal symbol of $\left(S_{k}\right)$.

The computation of the principal symbol is an immediate consequence of the previous theorems. To compute the subprincipal term, introduce a Toeplitz operator $\left(T_{k}\right)$ of $M_{r}$ whose principal symbol is the function $g_{0}$ defined by the integral in the statement of the corollary. Then by theorem 8.5, one has $S_{k} U_{k}-U_{k} T_{k}=k^{-1} R_{k}$ where 
$\left(R_{k}\right)$ is a Fourier integral operator with symbol $i^{*} f_{1}-\pi^{*} g_{1}$ and $g_{1}$ is the subprincipal symbol of $\left(T_{k}\right)$. Assume that $g_{1}$ is equal to the second integral in statement of the corollary so that the symbol of $\left(R_{k}\right)$ vanishes. Composing with $U_{k}^{*}$, it comes that

$$
U_{k}^{*} S_{k} U_{k}-T_{k}=k^{-1} U_{k}^{*} R_{k} .
$$

By theorem 8.4, $\left(U_{k}^{*} R_{k}\right)$ is a Toeplitz operator with vanishing principal symbol. Hence $\left(U_{k}^{*} S_{k} U_{k}\right)$ and $\left(T_{k}\right)$ have the same principal and subprincipal symbols.

8.6. Proof of theorem 8.3. The proof relies on some properties of Toeplitz operators proved in [3] that we recall now. First the Schwartz kernel of a Toeplitz operator is described in a similarly way as the one of a Fourier integral operators in section 8.4. The Schwartz kernel of a Toeplitz operator $\left(T_{k}\right)$ of $M_{r}$ is a sequence of holomorphic sections of

$$
\left(L_{r}^{k} \otimes \delta_{r}\right) \otimes\left(\bar{L}_{r}^{k} \otimes \bar{\delta}_{r}\right) \rightarrow M_{r} \times \bar{M}_{r},
$$

of the form

$$
\left(\frac{k}{2 \pi}\right)^{n_{r}} E_{r}^{k}(x, y) f(x, y, k)+O\left(k^{-\infty}\right),
$$

where $n_{r}$ is the complex dimension of $M_{r}, E_{r}$ and $f(\cdot, k)$ satisfy similar assumptions to those of section 8.4 with the moment Lagrangian replaced by the diagonal. Precisely, $E_{r}$ is a section of $L_{r} \otimes \bar{L}_{r} \rightarrow M_{r} \times \bar{M}_{r}$ whose restriction to the diagonal satisfies

$$
E_{r}(x, x)=u \otimes \bar{u}
$$

for any unitary vector $u \in L_{r, x}$. Furthermore $\bar{\partial} E_{r}$ vanishes to any order along the diagonal and outside the diagonal one has $\left\|E_{r}(x, y)\right\|<1 .(f(\cdot, k))$ is a sequence of sections of $\delta_{r} \otimes \bar{\delta}_{r} \rightarrow M_{r} \times \bar{M}_{r}$ which admits an asymptotic expansion in the $\mathcal{C}^{\infty}$ topology of the form

$$
f(., k)=f_{0}+k^{-1} f_{1}+k^{-2} f_{2}+\ldots
$$

whose coefficients satisfy $\bar{\partial} f_{i} \equiv 0$ modulo a section vanishing to any order along the diagonal. Finally we recover the principal symbol $h$ of the operator $\left(T_{k}\right)$ from the first coefficient $f_{0}$ by

$$
f_{0}(x, x)=h(x) t_{r}(x, x) .
$$

Here $t_{r}$ is the section of $\delta_{r} \otimes \bar{\delta}_{r}$ over the diagonal of $M_{r}^{2}$ such that

$$
t_{r}(x, x)=u \otimes \bar{u}
$$

if $u$ is a unitary vector of $\delta_{r, x}$.

Lemma 8.1. Let $\left(T_{k}\right)$ be a Toeplitz operator of $M_{r}$ with symbol $h$. Then

$$
\left(\frac{k}{2 \pi}\right)^{\frac{\ell}{4}} W_{k} T_{k}
$$

is a Fourier integral operator of $\mathcal{F}$ with symbol $\pi^{*} h$, where $\pi$ is the projection $P \rightarrow M_{r}$.

Theorem 8.3 follows. 
Proof. Recall that we denote by $\pi_{s}$ the projection from the stable set $M_{s}$ onto $M_{r}$. By definition of the Guillemin-Sternberg isomorphism, the Schwartz kernel of $W_{k} T_{k}$ over $M_{s} \times M$ is the pull-back of the Schwartz kernel of $T_{k}$ by the projection $\pi_{s} \times$ id. The result follows by comparing (44) and (45). We deduce from proposition 8.1, that the Schwartz kernel of $W_{k} T_{k}$ and its successive derivatives are $O\left(k^{-\infty}\right)$ on the complementary set of $\Lambda$. Next observe that we can choose the sections $E_{r}$ and $F$ in such a way that

$$
\left(\pi_{s} \otimes \mathrm{id}\right)^{*} E_{r}=F
$$

and the final result follows easily.

8.7. Proof of theorem 8.4. The Schwartz kernel of $Q_{k}^{*} R_{k}$ is given by the following integral

$$
\left(\frac{k}{2 \pi}\right)^{2 n_{r}+\frac{\ell}{2}} \int_{M} \bar{F}^{k}\left(y, z_{1}\right) \cdot F^{k}\left(y, z_{2}\right) f\left(y, z_{1}, z_{2}, k\right) \mu_{M}(y)
$$

where $f(\cdot, k)$ admits an asymptotic expansion in inverse power of $k$. The leading order satisfies

$$
f(x, z, z, k)=\overline{f_{Q}(x)} f_{R}(x) \overline{t_{\delta}(x, z)} \cdot t_{\delta}(x, z)+O\left(k^{-1}\right)
$$

for any $(x, z)$ in the moment Lagrangian, where $f_{Q}$ and $f_{R}$ are the symbols of $\left(Q_{k}\right)$ and $\left(R_{k}\right)$ respectively. We deduce from $(42),(43)$ and (46) that

$$
f(x, z, z, k)=\overline{f_{Q}(x)} f_{R}(x) 2^{\frac{\ell}{2}} \operatorname{det}^{-\frac{1}{2}}\left[g\left(\xi_{i}^{\#}, \xi_{j}^{\#}\right)\right](x) t_{r}(z, z)+O\left(k^{-1}\right) .
$$

Let us compute (48). First, since the pointwise norm of $F$ is smaller than 1 outside $\Lambda$, by modifying the integral by a $O\left(k^{-\infty}\right)$ if necessary, we may assume that the support of $f$ is a compact subset of $M_{r} \times M_{s} \times M_{r}$. So we just integrate on the stable set. Let us identify $M_{s}$ with $\mathfrak{g} \times P$ by the diffeomorphism (38). We will integrate successively on $\mathfrak{g}$, then on the fiber of $\pi: P \rightarrow M_{r}$ and finally on $M_{r}$. To do that, we write the Liouville measure on the stable set in the following way.

Lemma 8.2. We have over $\mathfrak{g} \times P$

$$
\mu_{M}(\xi, x)=\delta(\xi, x) \mu_{P}(x)\left|d t_{1} \ldots d t_{\ell}\right|(\xi)
$$

where $\mu_{P}$ is the invariant measure of $P$ whose push-forward by the projection $P \rightarrow$ $M_{r}$ is the Liouville measure $\mu_{M_{r}}$ of $M_{r}, t_{1}, \ldots, t_{\ell}$ are linear coordinates of $\mathfrak{g}$ in a orthogonal base $\xi_{1}, \ldots, \xi_{\ell}$ and $\delta \in \mathcal{C}^{\infty}(\mathfrak{g} \times P)$ satisfies

$$
\delta(0, x)=\operatorname{det}\left[g\left(\xi_{i}^{\#}, \xi_{j}^{\#}\right)\right](x), \quad \forall x \in P .
$$

This follows from the fact that the Riemannian volume of $G$ is 1 (cf. lemma 4.20 of [6] for a proof). Furthermore we deduce from (47) that for $y=\exp (i \xi) \cdot x$ with $x \in P$, one has

$$
\bar{F}^{k}\left(y, z_{1}\right) \cdot F^{k}\left(y, z_{2}\right)=e^{-k \varphi(\xi, x)} \bar{F}^{k}\left(x, z_{1}\right) \cdot F^{k}\left(x, z_{2}\right)
$$

with

$$
\varphi(\xi, x)=-\ln \left(\frac{\|\exp (i \xi) \cdot u\|^{2}}{\|u\|^{2}}\right), \quad \text { if } u \in L_{x} .
$$


By the Fubini theorem, the integral (48) is equal to

$$
\left(\frac{k}{2 \pi}\right)^{2 n_{r}} \int_{M_{r}} \bar{E}_{r}^{k}\left(z, z_{1}\right) \cdot E_{r}^{k}\left(z, z_{2}\right) f^{\prime \prime}\left(z, z_{1}, z_{2}, k\right) \mu_{M_{r}}(z)
$$

where $f^{\prime \prime}$ is the function of $M_{r}^{3}$ given by

$$
f^{\prime \prime}\left(z, z_{1}, z_{2}, k\right)=\int_{P_{z}} f^{\prime}\left(x, z_{1}, z_{2}\right) \mu_{P_{z}}(x)
$$

with $\mu_{P_{z}}$ the $G$-invariant measure of $P_{z}$ with total volume 1 . And $f^{\prime}$ is the function of $P \times M_{r}^{2}$ given by

$$
f^{\prime}\left(x, z_{1}, z_{2}, k\right)=\left(\frac{k}{2 \pi}\right)^{\frac{\ell}{2}} \int_{\mathfrak{g}} e^{-k \varphi(\xi, x)} f\left(\exp (i \xi) \cdot x, z_{1}, z_{2}, k\right) \delta(\xi, x)\left|d t_{1} \ldots d t_{\ell}\right|(\xi) .
$$

We estimate this integral by the stationary phase lemma. As we already saw in the proof of proposition 8.1, we have

$$
\partial_{t^{i}} \varphi(\xi, x)=-2 \mu^{\xi^{i}}(\exp (i \xi) \cdot x)
$$

and the critical set of $\varphi$ is $\{0\} \times P$. The second derivatives are

$$
\partial_{t^{j}} \partial_{t^{i}} \varphi(0, x)=2 g\left(\xi_{i}^{\#}, \xi_{j}^{\#}\right)(x) .
$$

Since this matrix is non-degenerate, $f^{\prime}$ has an asymptotic expansion in power of $k^{-1}$ with coefficients in $\mathcal{C}^{\infty}\left(P \times M_{r}^{2}\right)$. The first order term is given by

$$
f^{\prime}\left(x, z_{1}, z_{2}, k\right)=f\left(x, z_{1}, z_{2}, k\right) \delta(0, x) \operatorname{det}^{-\frac{1}{2}}\left[\partial_{t^{j}} \partial_{t^{i}} \varphi(0, x)\right]+O\left(k^{-1}\right) .
$$

By (51) and lemma 8.2, it follows that

$$
f^{\prime}\left(x, z_{1}, z_{2}, k\right)=f\left(x, z_{1}, z_{2}, k\right) 2^{-\frac{\ell}{2}} \operatorname{det}^{\frac{1}{2}}\left[g\left(\xi_{i}^{\#}, \xi_{j}^{\#}\right)\right](x)+O\left(k^{-1}\right) .
$$

By (49), we obtain for any $(x, z)$ in the momentum Lagrangian $\Lambda$ that

$$
f^{\prime}(x, z, z, k)=\overline{f_{Q}(x)} f_{R}(x) \mu_{P_{z}}(x) t_{r}(z, z)+O\left(k^{-1}\right) .
$$

Consequently

$$
f^{\prime \prime}(z, z, z, k)=\int_{P_{z}} \overline{f_{Q}(x)} f_{R}(x) \mu_{P_{z}}(x) t_{r}(z, z)+O\left(k^{-1}\right) .
$$

To end the proof we have to compute the integral (50). We recognize the integral corresponding to the composition of two Toeplitz operators (cf. [3]). So we obtain the Schwartz kernel of a Toeplitz operator whose principal symbol is the function $g$ such that

$$
f^{\prime \prime}(z, z, z, k)=g(z) t_{r}(z, z)+O\left(k^{-1}\right)
$$

which completes the proof. 
8.8. Proof of theorem 8.5. Let us sketch the proof. Consider a Fourier integral operator $\left(Q_{k}\right) \in \mathcal{F}$ and two Toeplitz operators $\left(S_{k}\right)$ and $\left(T_{k}\right)$ of $M$ and $M_{r}$ respectively. Then the Schwartz kernel of $S_{k} Q_{k}$ is the image of the Schwartz kernel of $Q_{k}$ by the map

$$
S_{k} \otimes \mathrm{id}: \mathcal{H}_{k} \otimes \overline{\mathcal{H}}_{r, k} \rightarrow \mathcal{H}_{k} \otimes \overline{\mathcal{H}}_{r, k}
$$

Similarly the Schwartz kernel of $Q_{k}$ is sent into the Schwartz kernel of $Q_{k} T_{k}$ by

$$
\operatorname{id} \otimes \overline{T_{k}^{*}}: \mathcal{H}_{k} \otimes \overline{\mathcal{H}}_{r, k} \rightarrow \mathcal{H}_{k} \otimes \overline{\mathcal{H}}_{r, k}
$$

$\left(S_{k} \otimes \mathrm{id}\right)$ and $\left(\mathrm{id} \otimes \overline{T_{k}^{*}}\right)$ are Toeplitz operators of $M \times \bar{M}_{r}$. The Schwartz kernel of $\left(Q_{k}\right)$ is a Lagrangian section of $M \times \bar{M}_{r}$ associated to the moment Lagrangian in the same way the eigenstates in section 5.1 are Lagrangian sections associated to the fiber of the integrable system. So we are reduced to consider the action of a Toeplitz operator on a Lagrangian section. This gives another Lagrangian section and in the favorable cases the subprincipal terms may be computed as it was explained in the paper [5], theorems 3.3 and 3.4. This will prove the theorem.

Let us apply this program. We denote by $p$ and $p_{r}$ the projection from $M \times M_{r}$ onto the first and second factor. Let $f_{0}, f_{1}$ and $g_{0}, g_{1}$ be the principal and subprincipal symbols of $\left(S_{k}\right)$ and $\left(T_{k}\right)$. Then it is easily seen that the principal and subprincipal symbols of $\left(S_{k} \otimes \mathrm{id}\right)$ (resp. $\left.\left(\mathrm{id} \otimes \overline{T_{k}^{*}}\right)\right)$ are $p^{*} f_{0}$ and $p^{*} f_{1}\left(\operatorname{resp} . p_{r}^{*} g_{0}\right.$ and $\left.p_{r}^{*} g_{1}\right)$.

We denote by $\left(\tilde{Q}_{k}\right)$ the sequence of the Schwartz kernels of $\left(Q_{k}\right)$. Let $h \in \mathcal{C}^{\infty}(P)$ be the symbol of the Fourier integral operator $\left(Q_{k}\right)$. Then the symbol of the Lagrangian section $\left(\tilde{Q}_{k}\right)$ is the section $\sigma$ of $\delta \otimes \bar{\delta}_{r} \rightarrow \Lambda$ given by

$$
(x, z) \in \Lambda \rightarrow \sigma(x, z)=h(x) t_{\delta}(x, z)
$$

where $t_{\delta}$ has been introduced in the beginning of section 8.4.

Consider a Toeplitz operator $\left(O_{k}\right)$ of $M \times \overline{M_{r}}$ with principal symbol $e_{0}$. Then by theorem 3.3 of [5] the symbol of $\left(O_{k} \tilde{Q}_{k}\right)$ is the product of $\sigma$ by the restriction of $e_{0}$ to the moment Lagrangian $\Lambda$. Applying this with the operator $O_{k}=S_{k} \otimes \overline{T_{k}^{*}}$, we deduce the first part of theorem 8.5. Indeed, since

$$
S_{k} \otimes \overline{T_{k}^{*}}=\left(S_{k} \otimes \mathrm{id}\right) \circ\left(\mathrm{id} \otimes \overline{T_{k}^{*}}\right),
$$

the principal symbol of $\left(O_{k}\right)$ is $\left(p^{*} f_{0}\right)\left(p_{r}^{*} g_{0}\right)$. Identifying $\mathcal{C}^{\infty}(\Lambda)$ with $\mathcal{C}^{\infty}(P)$, the restriction of this symbol to the moment Lagrangian becomes $\left(j^{*} f_{0}\right)\left(\pi^{*} f_{1}\right)$, where $j$ is the injection $P \rightarrow M$ and $\pi$ is the projection $P \rightarrow M_{r}$. So the symbol of the Fourier integral operator $\left(S_{k} Q_{k} T_{k}\right)$ is $\left(j^{*} f_{0}\right)\left(\pi^{*} f_{1}\right) h$. This proves the first assertion in theorem 8.5.

The proof of the second one is the difficult part. Assume that the restriction of the principal symbol $e_{0}$ to the moment Lagrangian vanishes. Then the symbol of $\left(O_{k} \cdot \tilde{Q}_{k}\right)$ vanishes and $\left(k O_{k} \tilde{Q}_{k}\right)$ is another Lagrangian section. By theorem 3.4 of [5], its symbol is equal to

$$
\left(i^{*} e_{1}+\frac{1}{i} \mathcal{L}_{X}^{\delta \otimes \overline{\delta_{r}}}\right) \sigma
$$

Here $e_{1}$ is the subprincipal symbol of $\left(O_{k}\right)$ and $i^{*} e_{1}$ its restriction to the momentum Lagrangian $\Lambda$. Since $e_{0}$ is constant over the Lagrangian manifold $\Lambda$, its Hamiltonian vector field is tangent to $\Lambda . X$ is defined as the restriction to $\Lambda$ of this Hamiltonian vector field. 
Finally the Lie derivative $\mathcal{L}_{X}^{\delta \otimes \overline{\delta_{r}}}$ has the following sense. On the one hand, $\delta \otimes \overline{\delta_{r}}$ is naturally a half-form bundle of $M \times \overline{M_{r}}$. Recall that we denoted by $\varphi$ the isomorphism $\delta^{2} \rightarrow \wedge^{\text {top }, 0} T^{*} M$ and by $\varphi_{r}$ the isomorphism $\delta_{r}^{2} \rightarrow \wedge^{\text {top }, 0} T^{*} M_{r}$. Then the map

$$
\varphi^{2} \otimes{\overline{\varphi_{r}}}^{2}: \delta^{2} \otimes{\overline{\delta_{r}}}^{2} \rightarrow \wedge^{\mathrm{top}, 0} T^{*} M \otimes \overline{\wedge^{\mathrm{top}, 0} T^{*} M_{r}} \simeq \wedge^{\mathrm{top}, 0} T^{*}\left(M \times \overline{M_{r}}\right)
$$

is an isomorphism of Hermitian holomorphic bundles. On the other hand the moment Lagrangian $\Lambda$ being a Lagrangian submanifold of $M \times M_{r}^{-}$, the pull-back by the embedding $i: \Lambda \rightarrow M \times M_{r}$ induces an isomorphism

$$
i^{*}: i^{*}\left(\wedge^{\mathrm{top}, 0} T^{*}\left(M \times \overline{M_{r}}\right)\right) \rightarrow \wedge^{\mathrm{top}} T^{*} \Lambda \otimes \mathbb{C} .
$$

Let $\varphi_{\Lambda}$ be the composition of these isomorphisms

$$
\varphi_{\Lambda}: i^{*}\left(\delta \otimes \overline{\delta_{r}}\right)^{2} \rightarrow \wedge^{\text {top }} T^{*} \Lambda \otimes \mathbb{C} .
$$

The Lie derivative $\mathcal{L}_{X}^{\delta \otimes \overline{\delta_{r}}}$ is then defined as the first order differential operator such that for any section $s$ of $i^{*}\left(\delta \otimes \overline{\delta_{r}}\right) \rightarrow \Lambda$, one has

$$
\mathcal{L}_{X} \varphi_{\Lambda}\left(s^{2}\right)=2 \varphi_{\Lambda}\left(s \otimes \mathcal{L}_{X}^{\delta \otimes \overline{\delta_{r}}} s\right)
$$

Actually we already considered such a Lie derivative in remark 5.2.

Let us apply this to the operator $O_{k}=S_{k} \otimes \mathrm{id}-\mathrm{id} \otimes \overline{T_{k}^{*}}$. Its principal symbol is $e_{0}=p^{*} f_{0}-p_{r}^{*} g_{0}$. Assume that $f_{0}$ is $G$-invariant and that $\pi^{*} g_{0}=j^{*} f_{0}$. Then the restriction of $e_{0}$ to $\Lambda$ vanishes. One has to compute the sum (52). First,

$$
e_{1}(x, z)=f_{1}(z)-g_{1}(x), \quad \forall(x, z) \in \Lambda .
$$

Second, identifying the momentum Lagrangian $\Lambda$ with the zero level set $P$, the restriction $X$ of the Hamiltonian vector field of $e_{0}$ to $\Lambda$ becomes the restriction $Y$ of the Hamiltonian vector field of $f_{0}$ to $P$. Then we will prove that

$$
\left(\mathcal{L}_{X}^{\delta \otimes \bar{\delta}} \sigma\right)(x, z)=\left(\mathcal{L}_{Y} h\right)(x) \cdot t_{\delta}(x), \quad \forall(x, z) \in \Lambda
$$

and theorem 8.5 follows directly. To show (53), we compute $\varphi_{\Lambda}\left(t_{\delta}^{2}\right)$.

Lemma 8.3. By identifying the momentum Lagrangian $\Lambda$ with $P, \varphi_{\Lambda}\left(t_{\delta}^{2}\right)$ is the volume element of $P$ such that

$$
\iota\left(\gamma_{\mathbb{R}}\right) \varphi_{\Lambda}\left(t_{\delta}^{2}\right)=i^{n_{r}\left(n_{r}-2\right)} \pi^{*} \omega_{r}^{\wedge n_{r}}
$$

where $n_{r}$ is the complex dimension of $M_{r}, \gamma_{\mathbb{R}}$ is the multivector $\xi_{1}^{\#} \wedge \ldots \wedge \xi_{\ell}^{\#}$ with $\left(\xi_{i}\right)$ an orthogonal base of $\mathfrak{g}$.

From this we deduce that the Lie derivative with respect to $X$ of $\varphi_{\Lambda}\left(t_{\delta}^{2}\right)$ vanishes and consequently the same holds for the Lie derivative of $t_{\delta}$, which proves (53).

Proof. By definition of $t_{\delta}$, if $x$ is a point of $P$ and $u \in \delta_{x}$ is such that the norm of $[u]$ is equal to 1 , then

$$
t_{\delta}(x, \pi(x))=u \otimes[\bar{u}] .
$$

By identifying the momentum Lagrangian with $P$, one has

$$
\varphi_{\Lambda}\left(t_{\delta}^{2}\right)=j^{*} \varphi\left(u^{2}\right) \wedge \pi^{*} \overline{\varphi_{r}\left([u]^{2}\right)},
$$


consequently,

$$
\iota\left(\gamma_{\mathbb{R}}\right) \varphi_{\Lambda}\left(t_{\delta}^{2}\right)=j^{*}\left(\iota\left(\gamma_{\mathbb{R}}\right) \varphi\left(u^{2}\right)\right) \wedge \pi^{*} \overline{\varphi_{r}\left([u]^{2}\right)} .
$$

Since $\varphi\left(u^{2}\right) \in \wedge^{\text {top }, 0} T^{*} M$, we have

$$
\iota\left(\gamma_{\mathbb{R}}\right) \varphi\left(u^{2}\right)=\iota(\gamma) \varphi\left(u^{2}\right)
$$

where $\gamma$ is the multivector defined in (40). Next by definition (41) of $\varphi_{r}$, we obtain that

$$
\iota\left(\gamma_{\mathbb{R}}\right) \varphi\left(u^{2}\right)=\pi_{s}^{*} \varphi_{r}\left([u]^{2}\right)
$$

Since $\pi=\pi_{s} \circ j$, it follows that

$$
j^{*}\left(\iota\left(\gamma_{\mathbb{R}}\right) \varphi\left(u^{2}\right)\right)=\pi^{*} \varphi_{r}\left([u]^{2}\right) .
$$

Hence,

$$
\iota\left(\gamma_{\mathbb{R}}\right) \varphi_{\Lambda}\left(t_{\delta}^{2}\right)=\pi^{*}\left(\varphi_{r}\left([u]^{2}\right) \wedge \overline{\varphi_{r}\left([u]^{2}\right)}\right)
$$

Because $[u]$ is unitary, $\varphi_{r}\left([u]^{2}\right)$ is also of norm 1 , we conclude.

\section{REFERENCES}

[1] D. Borthwick, T. Paul, And A. URIBe, Legendrian distributions with applications to relative Poincaré series, Invent. Math., 122:2 (1995), pp. 359-402.

[2] L. Boutet de Monvel and V. Gulllemin, The spectral theory of Toeplitz operators, volume 99 of Annals of Mathematics Studies, Princeton University Press, Princeton, NJ, 1981.

[3] L. Charles, Berezin-Toeplitz operators, a semi-classical approach, Comm. Math. Phys., 239:1-2 (2003), pp. 1-28.

[4] L. Charles, Quasimodes and Bohr-Sommerfeld conditions for the Toeplitz operators, Comm. Partial Differential Equations, 28:9-10 (2003), pp. 1527-1566.

[5] L. Charles, Symbolic calculus for Toeplitz operators with half-form, J. Symplectic Geom., 4:2 (2006), pp. 171-198.

[6] L. Charles, Toeplitz operators and Hamiltonian torus actions, J. Funct. Anal., 236:1 (2006), pp. 299-350.

[7] L. Charles, Semi-classical properties of geometric quantization with metaplectic correction, Comm. Math. Phys., 270:2 (2007), pp. 445-480.

[8] M. Farber, J.-C. Hausmann, and D. Schuetz, On the conjecture of kevin walker, 2007.

[9] V. Guillemin and S. SternberG, Geometric quantization and multiplicities of group representations, Invent. Math., 67:3 (1982), pp. 515-538.

[10] B. C. Hall And W. D. Kirwin, Unitarity in "quantization commutes with reduction", Comm. Math. Phys., 275:2 (2007), pp. 401-422.

[11] J.-C. Hausmann And A. Knutson, The cohomology ring of polygon spaces, Ann. Inst. Fourier (Grenoble), 48:1 (1998), pp. 281-321.

[12] L. Hörmander, The analysis of linear partial differential operators. I, volume 256 of Grundlehren der Mathematischen Wissenschaften [Fundamental Principles of Mathematical Sciences]. Springer-Verlag, Berlin, second edition, 1990. Distribution theory and Fourier analysis.

[13] L. C. Jeffrey and J. Weitsman, Bohr-Sommerfeld orbits in the moduli space of flat connections and the Verlinde dimension formula, Comm. Math. Phys., 150:3 (1992), pp. 593-630.

[14] M. Kapovich And J. J. Millson, The symplectic geometry of polygons in Euclidean space, J. Differential Geom., 44:3 (1996), pp. 479-513.

[15] A. A. KlyachKo, Spatial polygons and stable configurations of points in the projective line, in "Algebraic geometry and its applications (Yaroslavl', 1992)", Aspects Math., E25, pages 67-84. Vieweg, Braunschweig, 1994.

[16] J. Marché And M. NARImannejad, Some asymptotics of topological quantum field theory via skein theory, Duke Math. J., 141:3 (2008), pp. 573-587. 
[17] J. RoBerts, Classical 6j-symbols and the tetrahedron, Geom. Topol., 3 (1999), pp. 21-66 (electronic).

[18] R. SJAMAAR, Holomorphic slices, symplectic reduction and multiplicities of representations, Ann. of Math. (2), 141:1 (1995), pp. 87-129.

[19] Y. U. TAYLOR AND C. T. WoOdWARD, $6 j$ symbols for $U_{q}\left(\mathfrak{s l}_{2}\right)$ and non-Euclidean tetrahedra, Selecta Math. (N.S.), 11:3-4 (2005), pp. 539-571.

[20] V. G. Turaev, Skein quantization of Poisson algebras of loops on surfaces, Ann. Sci. École Norm. Sup. (4), 24:6 (1991), pp. 635-704.

[21] S. Vũ NGọc, Systèmes intégrables semi-classiques: du local au global, volume 22 of Panoramas et Synthèses [Panoramas and Syntheses], Société Mathématique de France, Paris, 2006. 